\section{Whole genome sequence analysis of blood lipid levels in $>66,000$ individuals}

Margaret Sunitha Selvaraj ${ }^{1,2,3}$, Xihao Li ${ }^{4}$, Zilin Li ${ }^{4}$, Akhil Pampana $^{2}$, David Y Zhang, ${ }^{5,6}$ Joseph Park $^{5,6}$, Stella Aslibekyan ${ }^{7}$, Joshua C Bis ${ }^{8}$, Jennifer A Brody ${ }^{8}$, Brian E Cade ${ }^{9}$, Lee-Ming Chuang ${ }^{10}$, Ren-Hua Chung ${ }^{11}$, Joanne E Curran ${ }^{12}$, Lisa de las Fuentes $^{13,14}$, Paul S de Vries ${ }^{15}$, Ravindranath Duggirala ${ }^{12}$, Barry I Freedman ${ }^{16}$, Mariaelisa Graff ${ }^{17}$, Xiuqing Guo ${ }^{18}$, Nancy Heard-Costa ${ }^{19}$, Bertha Hidalgo ${ }^{7}$, Chii-Min Hwu ${ }^{20}$, Marguerite R Irvin ${ }^{7}$, Tanika N Kelly ${ }^{21,22}$, Brian G Kral ${ }^{23}$, Leslie Lange ${ }^{24}$, Xiaohui Li ${ }^{18}$, Martin Lisa ${ }^{25}$, Steven A Lubitz ${ }^{1,26}$, Ani W Manichaikul ${ }^{27}$, Preuss Michael ${ }^{28}$, May E Montasser ${ }^{29}$, Alanna C Morrison $^{15}$, Take Naseri $^{30}$, Jeffrey R O'Connell ${ }^{29}$, Nicholette D Palmer ${ }^{31}$, Patricia A Peyser ${ }^{32}$, Muagututia S Reupena ${ }^{33}$, Jennifer A Smith ${ }^{32}$, Xiao Sun ${ }^{21}$, Kent D Taylor ${ }^{18}$, Russell P Tracy ${ }^{34}$, Michael Y Tsai ${ }^{35}$, Zhe Wang ${ }^{28}$, Yuxuan Wang ${ }^{36}$, Bao Wei $^{37}$, John T Wilkins ${ }^{38}$, Lisa R Yanek ${ }^{23}$, Wei Zhao ${ }^{32}$, Donna K Arnett ${ }^{39}$, John Blangero ${ }^{12}$, Eric Boerwinkle ${ }^{15}$, Donald W Bowden $^{31}$, Yii-Der Ida Chen ${ }^{40}$, Adolfo Correa ${ }^{41}$, L Adrienne Cupples ${ }^{36}$, Susan K Dutcher ${ }^{42}$, Patrick T Ellinor ${ }^{1,26}$, Myriam Fornage $^{43}$, Stacey Gabriel ${ }^{44}$, Soren Germer ${ }^{45}$, Richard Gibbs ${ }^{46}$, Jiang He ${ }^{21,22}$, Robert C Kaplan ${ }^{47,48}$, Sharon LR Kardia ${ }^{32}$, Ryan Kim ${ }^{49}$, Charles Kooperberg ${ }^{48}$, Ruth J. F. Loos ${ }^{28,50}$, Karine Martinez ${ }^{51}$, Rasika A Mathias ${ }^{23}$, Stephen T McGarvey ${ }^{52}$, Braxton D Mitchell ${ }^{29,53}$, Deborah Nickerson ${ }^{54}$, Kari E North ${ }^{17}$, Bruce M Psaty ${ }^{8,55,56}$, Susan Redline ${ }^{9}$, Alexander P Reiner ${ }^{55,48}$, Ramachandran S Vasan ${ }^{57,58,59}$, Stephen S Rich ${ }^{27}$, Cristen Willer ${ }^{60}$, Jerome I Rotter ${ }^{18}$, Daniel J Rader ${ }^{5,6,61}$, Xihong Lin ${ }^{2,4,62}$, NHLBI Trans-Omics for Precision Medicine (TOPMed) Consortium, Gina M Peloso ${ }^{36 \# \$}$, Pradeep Natarajan $^{1,2,3} \# \$$

1. Cardiovascular Research Center, Massachusetts General Hospital, Boston, MA, USA, 02114

2. Program in Medical and Population Genetics, Broad Institute of Harvard and MIT, Cambridge, MA, USA, 02142

3. Department of Medicine, Harvard Medical School, Boston, MA, USA, 02115

4. Department of Biostatistics, Harvard T.H. Chan School of Public Health, Boston, MA, USA, 02115

5. Department of Genetics, Perelman School of Medicine, University of Pennsylvania, Philadelphia, PA, USA, 19104

6. Department of Medicine, Perelman School of Medicine, University of Pennsylvania, Philadelphia, PA, USA, 19104

7. Department of Epidemiology, University of Alabama at Birmingham School of Public Health

8. Cardiovascular Health Research Unit, Department of Medicine, University of Washington, Seattle, WA 
9. Department of Medicine, Brigham and Women's Hospital, Harvard Medical School

10. Department of Internal Medicine, National Taiwan University Hospital, Taipei, Taiwan

11. Institute of Population Health Sciences, National Health Research Institutes, Zhunan, 350, Taiwan

12. Department of Human Genetics and South Texas Diabetes and Obesity Institute, University of Texas Rio Grande

Valley School of Medicine, Brownsville, TX 78520

13. Department of Medicine, Cardiovascular Division, Washington University School of Medicine, St. Louis, MO

14. Division of Biostatistics, Washington University School of Medicine, St. Louis, MO

15. Human Genetics Center, Department of Epidemiology, Human Genetics, and Environmental Sciences, School of Public Health, The University of Texas Health Science Center at Houston, Houston, Texas, USA

16. Department of Internal Medicine, Section on Nephrology, Wake Forest School of Medicine, Winston-Salem, NC, USA, 27157

17. Department of Epidemiology, UNC Chapel Hill

18. The Institute for Translational Genomics and Population Sciences, Department of Pediatrics, The Lundquist Institute for Biomedical Innovation at Harbor-UCLA Medical Center, Torrance, CA USA

19. Department of Neurology, Boston university School of Medicine

20. Section of Endocrinology and Metabolism, Department of Medicine, Taipei Veterans General Hospital

21. Department of Epidemiology, Tulane University School of Public Health and Tropical Medicine, New Orleans, Louisiana, US, 70112

22. Tulane University Translational Science Institute, New Orleans, Louisiana, US, 70112

23. Department of Medicine, Johns Hopkins University School of Medicine, Baltimore, MD, USA 21206

24. Division of Biomedical Informatics and Personalized Medicine, Department of Medicine

25. Department of Medicine, George Washington University, Washingron DC

26. Cardiovascular Disease Initiative, The Broad Institute of MIT and Harvard, Cambridge, MA 02124

27. Department of Public Health Sciences, Center for Public Health Genomics, University of Virginia, Charlottesville, VA USA

28. The Charles Bronfman Institute for Personalized Medicine, Icahn School of Medicine at Mount Sinai, New York, NY

29. Department of Medicine, University of Maryland School of Medicine, Baltimore, MD

30. Ministry of Health, Government of Samoa, Samoa

31. Department of Biochemistry, Wake Forest School of Medicine, Winston-Salem, NC, USA, 27157

32. Department of Epidemiology, University of Michigan, Ann Arbor, MI, USA 48109

33. Lutia i Puava ae Mapu i Fagalele, Apia, Samoa

34. Departments of Pathology \& Laboratory Medicine and Biochemistry, Larner College of Medicine at the University of Vermont, Colchester, VT USA 
35. Department of Laboratory Medicine and Pathology, University of Minneosta, Minneapolis, MN USA 36. Department of Biostatistics, Boston University School of Public Health, Boston, MA, USA, 02118

37. Department of Epidemiology, Universiyy of lowa

38. Department of Medicine (Cardiology) and Department of Preventive Medicine, Northwestern University Feinberg School of Medicine, Chicago, IL

39. Dean's Office, University of Kentucky College of Public Health

40. Lundquist Institute for Biomedical Innovation at Harbor-UCLA Medical Center

41. Department of Population Health Science, University of Mississippi Medical Center

42. McDonnell Genome Institute

43. Brown Foundation Institute of Molecular Medicine, McGovern Medical School, The University of Texas Health Science Center at Houston, Houston, Texas, 77225

44. Broad Institute, Cambridge, Massachusetts, 02142

45. New York Genome Center, New York, New York, 10013

46. Baylor College of Medicine Human Genome Sequencing Center, Houston, Texas, 77030

47. Department of Epidemiology and Population Health, Albert Einstein College of Medicine, Bronx NY 10461 USA

48. Division of Public Health Sciences, Fred Hutchinson Cancer Research Center, Seattle WA 98109

49. Psomagen

50. NNF Center for Basic Metabolic Research, University of Copenhagen, Cophenhagen, Denmark

51. Illumina

52. Department of Epidemiology, International Health Institute, Brown University, Providence RI

53. Geriatrics Research and Education Clinical Center, Baltimore Veterans Administration Medical Center, Baltimore, MD

54. University of Washington, Department of Genome Sciences, Seattle, Washington, 98195

55. Department of Epidemiology, University of Washington, Seattle, WA

56. Department of Health Services, University of Washington, Seattle, WA

57. Sections of Preventive medicine and Epidemiology, Cardiovascular medicine, Department of Medicine, Boston University School of Medicine

58. Department of Epidemiology, Boston University School of Public Health

59. Framingham Heart Study

60. University of Michigan, Internal Medicine, Ann Arbor, Michigan, 48109

61. Institute for Translational Medicine and Therapeutics, Perelman School of Medicine, University of Pennsylvania, Philadelphia, PA, USA, 19104

62. Department of Statistics, Harvard University, Cambridge, MA, USA, 02138 


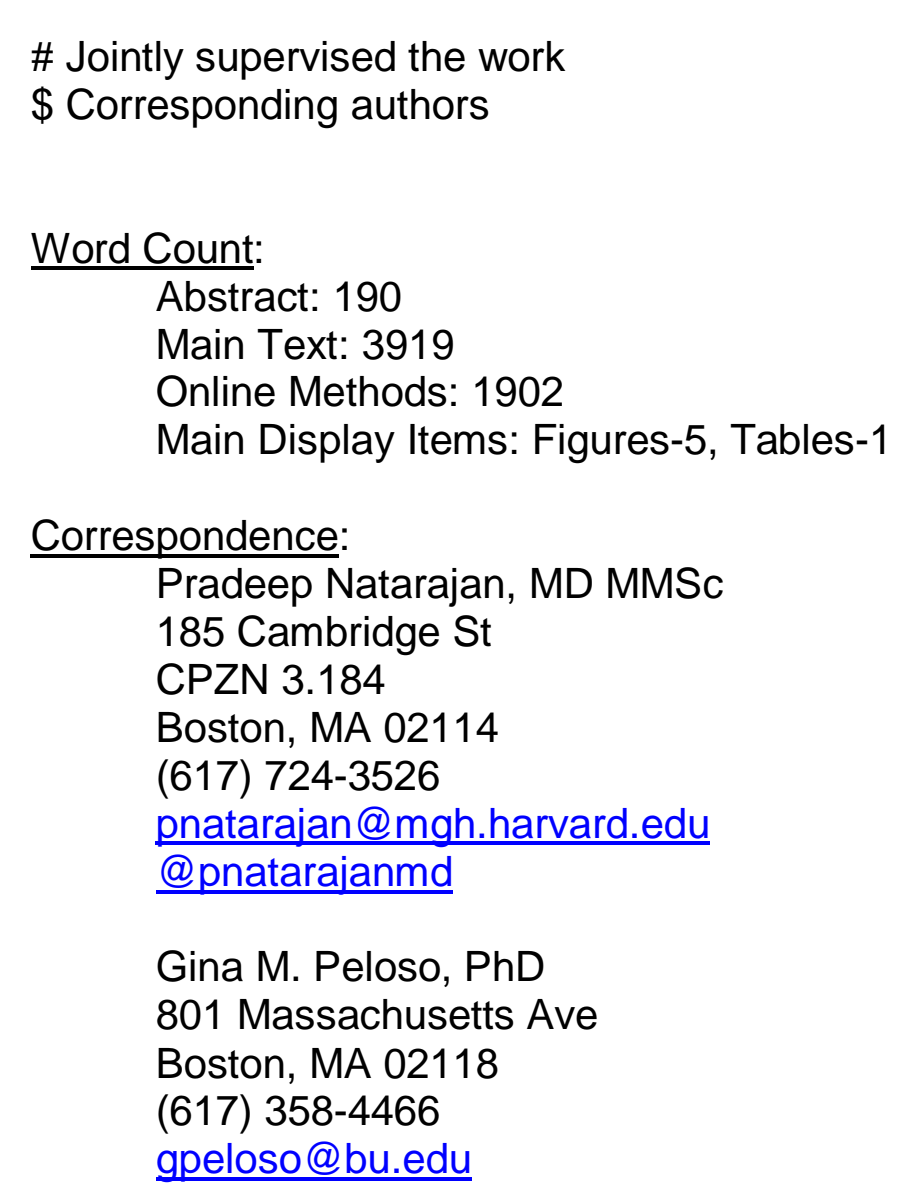




\section{Abstract:}

119 Plasma lipids are heritable modifiable causal factors for coronary artery disease, the leading cause of death globally.

120 Despite the well-described monogenic and polygenic bases of dyslipidemia, limitations remain in discovery of lipid-

121 associated alleles using whole genome sequencing, partly due to limited sample sizes, ancestral diversity, and

122 interpretation of potential clinical significance. Increasingly larger whole genome sequence datasets with plasma lipids

123 coupled with methodologic advances enable us to more fully catalog the allelic spectrum for lipids. Here, among 66,329

124 ancestrally diverse (56\% non-European ancestry) participants, we associate 428M variants from deep-coverage whole

125 genome sequences with plasma lipids. Approximately 400M of these variants were not studied in prior lipids genetic

126 analyses. We find multiple lipid-related genes strongly associated with plasma lipids through analysis of common and rare

127 coding variants. We additionally discover several significantly associated rare non-coding variants largely at Mendelian

128 lipid genes. Notably, we detect rare $L D L R$ intronic variants associated with markedly increased LDL-C, similar to rare

$129 L D L R$ exonic variants. In conclusion, we conducted a systematic whole genome scan for plasma lipids expanding the

130 alleles linked to lipids for multiple ancestries and characterize a clinically-relevant rare non-coding variant model for lipids. 


\section{Introduction}

Discovery of rare alleles linked to plasma lipids (i.e., low-density lipoprotein cholesterol [LDL-C], high-density lipoprotein cholesterol [HDL-C], total cholesterol [TC], and triglycerides [TG]) continue to yield important translational insights toward coronary artery disease (CAD), including PCSK9 and ANGPTL3 inhibitors now available in clinical practice $^{1,2,3,4,5}$. The monogenic and polygenic bases of plasma lipids are well-suited to population-based discovery analyses and confer broader insights for genetic analyses of complex traits. We now evaluate numerous newly catalogued, largely rare, alleles never previously systematically analyzed with lipids.

Analyses of imputed array-derived genome-wide genotypes and whole exome sequences in hundreds of thousands of increasingly diverse individuals continue to uncover low-frequency protein-coding variants linked to lipids.

Due to purifying selection, causal variants conferring large effects tend to occur relatively more recently, and are thus rare and often specific to families or communities ${ }^{6}$. Most discovery analyses for large-effect rare alleles have focused on the analysis of disruptive protein-coding variants given (1) well-recognized constraint in coding regions, (2) incomplete genotyping of rare non-coding sequence given relative sparsity of deep-coverage (i.e., $>30 X)$ whole genome sequencing (WGS), and (3) better prediction of coding versus non-coding sequence variation consequence ${ }^{1,7,8,9,10,11,12}$. We recently described a statistical framework incorporating multi-dimensional reference datasets paired with genomic data to improve rare coding and non-coding variant analyses for WGS analysis of lipids and other complex traits ${ }^{13,14}$. Furthermore, 
147 including individuals of non-European ancestry facilitates the discovery of both novel alleles at established loci as well as

148 novel loci $14,15,16$

149 Here, we examine the full allelic spectrum with plasma lipids using whole genome sequences and harmonized

150 lipids from the National Heart, Lung, and Blood Institute (NHLBI) Trans-Omics for Precision Medicine (TOPMed)

151 program ${ }^{17,18}$. We studied 66,329 participants and 428 million variants across multiple ancestry groups $-44.48 \%$

152 European, 25.60\% Black, 21.02\% Hispanic, 7.11\% Asian and 1.78\% Samoan. We identified robust allelic heterogeneity at

153 known loci with several novel variants at these loci; we additionally identified novel loci and pursued replication in

154 independent cohorts (31.50\% non-European samples). We then explored the association of genome-wide rare variants

155 with lipids, with detailed explorations of rare coding and non-coding variant models at known Mendelian dyslipidemia

156 genes. Our systemic effort yields new insights for plasma lipids provides a framework for population based WGS analysis

157 of complex traits.

158

159 Results

\section{Overview}

161 We studied the TOPMed Freeze8 dataset of 66,329 samples from 21 studies and performed genome-wide

162 association studies (GWAS) separately for the four plasma lipid phenotypes (i.e., LDL-C, HDL-C, TC and TG) using 28M

163 individual autosomal variants (minor allele count [MAC] > 20) and aggregated rare autosomal variant (minor allele 
164 frequency $[\mathrm{MAF}]<1 \%$ ) association testing for $417 \mathrm{M}$ variants (Fig. 1, Supplementary Fig. 1). Secondarily, we associated

165 individual variants with minor allele frequencies (MAF) $>0.01 \%$ within each ancestry group to detect ancestry-specific

166 lipid-associated alleles. We intersected our results with currently published array-based GWAS results ${ }^{15}$ to identify novel

167 associations with lipids. We performed replication analyses for the putative novel associations identified, in up to

168 approximately 45,000 independent samples with array-based genotyping imputed to TOPMed. Finally, we conducted rare

169 variant association studies as multiple aggregate tests across the genome to identify gene-specific functional categories

170 and non-coding genomic regions influencing plasma lipid concentrations.

171

172 TOPMed baseline characteristics

173 The TOPMed Informatics Research Center (IRC) and TOPMed Data Coordinating Center (DCC) performed quality 174 control, variant calling, and calculated the relatedness of population structures of Freeze 8 data ${ }^{17}$. We studied 66,329

175 samples across 21 cohorts and 41,182 (62\%) were female. The ancestry distribution was 29,502 (44.46\%) White, 16,983

176 (25.60\%) Black, 13,943 (21.02\%) Hispanic, 4,719 (7.11\%) Asian, and 1,182 (1.78\%) Samoan (Supplementary Table 1).

177 The mean (standard deviation [SD]) age of the full cohort was 53 (15.00) years which varied by cohort from 25 (3.56)

178 years for Coronary Artery Risk Development in Young Adults (CARDIA) to 73 (5.38) years for Cardiovascular Health

179 Study (CHS). The Amish cohort had a higher-than-average concentration of LDL-C (140 [SD 43] mg/dL) and HDL-C (56

180 [SD 16] mg/dL) as well as lower TG (median 63 [IQR 50] mg/dL) consistent with the known founder mutations in $A P O B$ 
181 and $A P O C 3^{7,8,14}$. In the Women's Health Initiative (WHI) cohort, the TC (230 [SD 41] mg/dL) and TG (median 129 [IQR

$18287] \mathrm{mg} / \mathrm{dL}$ ) concentrations were higher than for other cohorts as previously described ${ }^{12}$. We accounted for lipid-lowering

183 medications and fasting status and inverse rank normalized the phenotypes as before ${ }^{12,14}$ which are further detailed in the

184 Methods. The adjusted normalized lipid concentrations for the four lipids were similar across the cohorts.

185 A total of $428 \mathrm{M}$ variants passed the quality criteria with an average depth $>30 \mathrm{X}$ in 22 autosomes. $202 \mathrm{M}$ variants

186 were singletons, $417 \mathrm{M}$ were rare variants $(\mathrm{MAF}<1 \%)$, and $11 \mathrm{M}$ were common or low frequency variants $(\mathrm{MAF}>1 \%)$ with

187 differences by cohort (Supplementary Table 2).

188

189 Individual variant associations with lipids

190 Approximately $28 \mathrm{M}$ variants with $\mathrm{MAC}>20$ were individually associated with LDL-C, HDL-C, TC and TG. We used

$191 \mathrm{p}$-value $<5 \times 10^{-9}$ to claim significance as previously recommended for whole genome sequencing common variant

192 association studies ${ }^{14,19}$. The total numbers of variants that met our significance threshold were 2,214, 2,314, 2,697 and

1932,442 for LDL-C, HDL-C, TC and TG, respectively, and after clumping ${ }^{20}$ the numbers of variants were $357,338,324$, and

194289 , respectively. Of these variants, most were previously demonstrated to be associated with plasma lipids either at the

195 variant- or locus-level ${ }^{15}$ (Supplementary Table 3, Supplementary Fig. 2).

196 To identify putative novel variant associations, we compared our results to a recent multi-ethnic lipid GWAS among

197312,571 participants of the Million Veteran Program (MVP) ${ }^{15}$ as well as the GWAS Catalog (All associations(v1.0) file 
198 dated 06/04/2020) (Fig. 2). We clumped (window $250 \mathrm{~kb}, \mathrm{r}^{2}$ 0.5) significant variants using Plink ${ }^{20}$ and queried these in the

199 GWAS Catalog and MVP. Among genome-wide significant variants, we tabulated 'known-position' (variant previously

200 associated), 'known-loci' (variants not previously significantly associated with the corresponding lipid phenotype but within

$201500 \mathrm{~kb}$ of a known locus, thereby representing additional allelic heterogeneity), and 'novel' variants (variants not in a

202 known lipid locus) (Supplementary Table 3).

203 The novel variants, tabulated in Table 1, are divided into two subsets - 'novel variants' or variants at established

204 lipid loci for another lipid phenotype, and 'novel loci,' representing new loci associations for any lipid phenotype. For

205 example, the CETP locus is well-known for its link to HDL-C, but we now found that rs 183130 (16:56957451:C:T, MAF

$20628.3 \%)$ at the locus is associated with LDL-C. Similarly, the variants rs7140110 (13:113841051:T:C, MAF 27.8\%) GAS6

207 and rs73729083 (7:137875053:T:C, MAF 4.5\%) CREB3L2 are newly associated with TC, while previous studies showed

208 that rs73729083 associates with LDL-C ${ }^{21}$ and rs7140110 associates with LDL-C ${ }^{22}$ and TG ${ }^{23}$. Index variants at novel loci

209 were typically low frequency variants often observed in non-European ancestries, so we also conducted ancestry-specific

210 association analyses for these alleles (Supplementary Table 4). For example, 12q23.1 (12:97352354:T:C, MAF 0.3\%)

211 and 4q34.2 (4:176382171:C:T, MAF 0.2\%) associations with LDL-C are specific to Hispanic (MAF 1.3\%) and Black (MAF

$2120.6 \%$ ) populations, respectively and among Asians (MAF 1.5\%) alone, 11q13.3 (11:69219641:C:T, MAF 0.2\%) was

213 associated with TG. One variant initially passing the novel locus filter for HDL-C (RNF111 - rs 112147665 , beta $=8.664$, $\mathrm{p}-$

214 value $\left.=6.51 \times 10^{-10}\right)$, was in LD $(r=0.7)$ with LIPC p.Thr405Met ( $r$ 113298164) which is known to be associated with HDL- 
215 C. The lead variant from MVP was $604 \mathrm{~kb}$ away from the RNF111 variant but the rare LIPC missense variant p.Thr405Met

216 was $421 \mathrm{~kb}$ away. Conditional analysis accounting for LIPC p.Thr405Met rendered the non-coding variant near RNF111

217 variant non-significant (beta $=4.351, \mathrm{p}$-value $=2.47 \times 10^{-02}$ ), therefore we reclassified $R N F 111$ variant as a known-position

218 variant. Ancestry-specific GWAS did not yield additional novel loci beyond our larger trans-ancestry GWAS. Majority of

219 genome significant single variants were captured by previous lipid GWAS ${ }^{15}$, but ancestry specific novel-hits are unique to

220 WGS TOPMed data.

221 Due to the paucity of available diverse WGS datasets with lipids of comparable size, we pursued replication with

222 two genome-wide array-based genotyped datasets imputed to TOPMed WGS ${ }^{17,24}$ : Mass General Brigham (MGB)

223 Biobank $(N=25,137)$ and Penn Medicine Biobank $(N=20,079)^{25,26}$, the replication cohorts had diverse ancestry distribution,

224 where non-European samples accounted for 15.77\% in MGB Biobank and 51.20\% in Penn Medicine Biobank

225 (Supplementary Table 5). We brought seven putative novel variants with $p$-values $<5 \times 10^{-9}$ forward for replication. The

226 three common variants, rs183130 (CETP), rs7140110 (GAS6) and rs73729083 (CREB3L2), that were associated with

227 both LDL-C and TC in TOPMed also replicated in MGB and two (rs183130, rs73729083) replicated in Penn Biobank at an

228 alpha level of 0.05 and consistent direction of effect (Table 1). The two variants that were associated in both replication

229 studies were most significantly associated among African Americans in TOPMed (rs183130: beta $=-2.762 \mathrm{mg} / \mathrm{dL}$, p-value

$230=5.71 \times 10^{-07} ; \mathrm{rs} 73729083:$ beta $=-3.725 \mathrm{mg} / \mathrm{dL}$, $\mathrm{p}$-value $\left.=5.25 \times 10^{-07}\right)$. Low-frequency variants from specific ancestry

231 groups associated with lipids in TOPMed were not replicated but we cannot rule out the possibility of reduced power due 
232 to general underrepresentation of non-white ancestry groups in the replication data. In exploratory analyses, we extended

233 the same approach for variants discovered to have $5 \times 10^{-9}<\mathrm{p}$-value $<5 \times 10^{-7}$ but did not observe replication

\section{4 (Supplementary Table 6).}

\section{CETP locus, HDL-C, and LDL-C}

CETP is a well-recognized Mendelian HDL-C gene and the locus was previously known to be significantly

238 associated with HDL-C, TC and TG at genome-wide significance ${ }^{15}$. Pharmacologic CETP inhibitors have shown strong

239 associations with increased HDL-C but mixed effects for LDL-C reduction in clinical trials ${ }^{27,28,29,30}$. We found that the CETP

240 locus variant rs183130 (chr16:56957451:C:T, MAF 28.3\%, intergenic variant) was associated with reduced LDL-C

241 concentration (beta $=-1.568 \mathrm{mg} / \mathrm{dL}, \mathrm{SE}=0.264, \mathrm{p}$-value $=2.88 \times 10^{-09}$ ). The lead HDL-C-associated variant at the locus,

242 rs3764261 (chr16:56959412:C:A, MAF 30.3\%), was associated with $3.5 \mathrm{mg} / \mathrm{dL}$ increased HDL-C (p-value = 8.03x10 ${ }^{-283}$ ),

243 and rs 183130 was associated with $3.9 \mathrm{mg} / \mathrm{dL}$ increased $\mathrm{HDL}-\mathrm{C}$ ( $\mathrm{p}$-value $<1 \times 10^{-284}$ ) as well. Among the ancestry groups

244 analyzed, rs183130 was most significantly associated with LDL-C among those of African ancestry (beta = $-2.762 \mathrm{mg} / \mathrm{dL}$,

$245 \mathrm{p}$-value $\left.=5.71 \times 10^{-07}\right)($ Supplementary Table 7). We next investigated variants by their HDL-C and LDL-C effects within

246 this locus (+/- 500kb of rs183130 and rs3764261) (Fig. 3). We identified five variants showing at least suggestive (p-value

$247<5 \times 10^{-07}$ ) association with both HDL-C and LDL-C. Though variants with strong LD (linkage disequilibrium) existed,

248 ancestry-specific analyses showed that the stronger LDL-C effects were among those of African ancestry. 
250 with eQTLs from 3 tissues (Liver, Adipose Subcutaneous and Adipose Visceral [Omentum]) from GTEx ${ }^{31}$. We analyzed 5

251 LDL-C and $441 \mathrm{HDL}-\mathrm{C}$ associated ( $\mathrm{p}$-values $<5 \times 10^{-07}$ ) variants. We correlated eQTL effect estimates for genes at the

252 locus with lipid outcome effect estimates. Indeed, CETP gene expression effects were strongly negatively correlated with

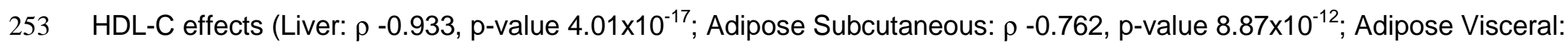

$254 \rho-0.739$, p-value 5.52 $\times 10^{-10}$ ) (Supplementary Fig. 3). However, CETP expression effects were not significantly

255 correlated with LDL-C (Liver: $\rho$ 0.007, p-value 0.99; Adipose Subcutaneous: $\rho$ 0.344, p-value 0.57; Adipose Visceral: $\rho$ -

$2560.59, \mathrm{p}$-value 0.29). Given the possibility that the observed lack of correlation for LDL-C could be due to reduced power

257 from a limited number of variants attaining a suggestive $p$-value $\left(<5 \times 10^{-07}\right)$, we repeated the analysis with a subset of 122

258 nominally significant ( $p$-value $<0.05$ ) LDL-C associated variants in this locus. Indeed, CETP gene expression effects were

259 strongly positively correlated with LDL-C effects (Liver: $\rho$ 0.957, $p$-value 2.28x10-08; Adipose Subcutaneous: $\rho$ 0.922, p-

260 value $1.34 \times 10^{-15}$; Adipose Visceral: $\rho$ 0.868, $p$-value $6.09 \times 10^{-11}$ ).

261

262 Rare variant aggregates associated with lipids

263 I) Gene-Centric associations

264 We next evaluated the association of aggregated rare (MAF<1\%) variants, linked to protein-coding genes ('gene-

265 centric'). We employed a Bonferroni-corrected significance threshold of $0.05 / 20,000=2.5 \times 10^{-06}$ for coding and non-coding 
gene-centric rare variant analyses (Supplementary Fig. 4). We identified 102 coding and 160 non-coding gene-centric

267 rare variant aggregates significantly associated with at least one of the four plasma lipid phenotypes in nonconditional

268 analysis (Supplementary Table 8-9). We secondarily conditioned our significant aggregate sets on variants individually

269 associated with lipid levels from the GWAS catalog, MVP summary statistics and the TOPMed data. We identified 74

270 coding and 25 non-coding rare variants aggregates associated with at least one lipid level after conditional analyses

\section{1 (Supplementary Table 10-11).}

272 Most of the coding gene-centric sets remained significant after secondary conditioning while a minority of non-

273 coding gene-centric sets remained significant after conditioning. Significant genes identified from coding rare variant

274 analyses included multiple known Mendelian lipid genes including $L C A T, L D L R$, and $A P O B$ (Supplementary Table 10).

$275 R F C 2$ putative loss-of-function mutations (combined allele frequency $<0.002 \%$ ) were significantly associated with

276 triglycerides ( $\mathrm{p}$-value $2 \times 10^{-06}$ ) representing a putative novel association for triglycerides. The RFC2 aggregate set (plof)

277 was associated with reduced TG (beta $=-0.89$ for $\log [\mathrm{TG}]$ ). The persistently significant regions identified from non-coding

278 rare variant analyses linked to genes included the UTR (untranslated region) for CETP and promoter-CAGE (CAGE- Cap

279 Analysis of Gene Expression sites) around APOA1 for HDL-C, and APOE promoter-CAGE, APOE enhancer-DHS (DHS -

280 DNase hypersensitivity sites), and EHD3 promoter-DHS for total cholesterol (Supplementary Table 11). Most of the

281 coding aggregates had larger effects compared to non-coding aggregates, and among the non-coding aggregates SPC24 
non-coding aggregate (enhancer-CAGE) at the $L D L R$ locus had the strongest effect for LDL-C (beta $=2.320 \mathrm{mg} / \mathrm{dL} ; \mathrm{p}$ value $\left.=1.75 \times 10^{-05}\right)$

\section{II) Region-Based associations}

We also performed unbiased region-based rare variant association analyses tiled across the genome with both static and dynamic window sizes. We first evaluated $2.6 \mathrm{M}$ regions statically at $2 \mathrm{~kb}$ size and $1 \mathrm{~kb}$ window overlap by the sliding window approach. Statistical significance was assigned at $0.05 /\left(2.6 \times 1^{-06}\right)=1.88 \times 10^{-08}$. We identified 28 significantly associated windows with at least one lipid phenotype. After conditioning on variants individually associated with the corresponding lipid phenotype, we identified two regions at $L D L R$ still significantly associated with both total cholesterol and LDL-C although these regions included both intronic and exonic variants (Supplementary Table 12). $L D L R$ intron 1 , which encodes LDLR-AS1 (LDLR antisense RNA 1) on the minus strand, had suggestive evidence for association with TC $\left(\mathrm{p}\right.$-value $\left.3.17 \times 10^{-6}\right)$ with $-2.76 \mathrm{mg} / \mathrm{dL}$ reduction in TC. A prior study identify that a common variant (rs6511720, MAF 0.11 ) in $L D L R$ intron 1 is associated with increased $L D L R$ expression in a luciferase assay and reduction in $L D L-C^{32}$. When adjusting for rs6511720, the significance improved ( $\mathrm{p}$-value $1.43 \times 10^{-8}$ ) with $-3.35 \mathrm{mg} / \mathrm{dL}$ reduction in TC.

For dynamic window scanning of the genome, we implemented the SCANG method ${ }^{33}$. The SCANG procedure accounts for multiple testing by controlling the genome-wide error rate (GWER) at $0.1^{33}$. In the dynamic window-based workflow, STAAR-O detected 51 regions significantly associated with at least one lipid phenotype after conditioning on 
299 known variants (Supplementary Table 13). Most of the regions mapped to known Mendelian lipid genes, including $L C A T$

$300\left(8.7 \times 10^{-13}\right)$ for HDL-C, and $\operatorname{LDLR}\left(2.4 \times 10^{-28}, 7.3 \times 10^{-26}\right)$ and PCSK9 $\left(2.9 \times 10^{-12}, 5.5 \times 10^{-12}\right)$ for LDL-C and TC, respectively.

301 Exon 4 aggregates of $L D L R$ were specifically associated with $20 \mathrm{mg} / \mathrm{dL}$ increase in LDL-C. PCSK9 Exon2-Intron2 region

302 spanning chr1:55043782-55045960 had significantly reduced LDL-C by $6 \mathrm{mg} / \mathrm{dL}\left(\mathrm{p}-\mathrm{value}=3 \times 10^{-13}\right.$ ), and the effect

303 persisted even with only Intron 2 rare variants of PCSK $9\left(-5 \mathrm{mg} / \mathrm{dL}, \mathrm{p}\right.$-value $\left.=2 \times 10^{-8}\right)$. Strikingly, in secondary analyses,

304 we found evidence for very large effects for rare variants in $L D L R$ Introns 2 and $3\left(+21 \mathrm{mg} / \mathrm{dL}, \mathrm{p}-\mathrm{value}=7 \times 10^{-4}\right)$ and

$305 L D L R$ Introns 16 and $17(+17 \mathrm{mg} / \mathrm{dL}$, $\mathrm{p}$-value $=0.02)$, similar to rare coding $L D L R$ mutations. While 32 of the significant

306 dynamic windows also included exonic regions, there were also several dynamic windows significantly independently

307 associated with lipids not containing exonic regions. For example, four non-coding windows (two overlapping) at 2 p24.1,

308 which harbors the Mendelian $A P O B$ gene, were significantly associated with LDL-C. Intronic non-coding regions were

309 associated with both LDL-C and TC -associated windows at LPAL2-LPA-SLC22A3; for example LPAL2 Intron 3 was

310 associated with a $3.7 \mathrm{mg} / \mathrm{dL}$ increase in TC. Non-coding TC-associated significant dynamic windows were near

311 TOMM40/APOE. One rare variant signal observed was at TOMM40 Intron 6, where the 'poly-T' variant in this region is on

312 the APOE4 haplotype and influences expressivity for Alzheimer's disease age-of-onset ${ }^{34,35}$. For HDL-C, we identified

313 significant non-coding windows at an intergenic region near $L P L$ and $C D 36$ Intron 4 . In the generation of the

314 spontaneously hypertensive rat model, the deletion of intron 4 in Cd36 with resultant Cd36 deficiency has been mapped to 
315 defective fatty acid metabolism in this model ${ }^{36}$. Several regions significant in SCANG were not even nominally significant

316 in burden association analyses indicating the likelihood of causal variants with bidirectional effects.

317 Several gene-centric non-coding aggregates associated with lipids near known monogenic lipid genes but mapped

318 to another gene at the locus via annotations. Therefore, we performed downstream conditional analyses adjusting the

319 gene-centric non-coding results for rare coding variants (MAF<1\%) within known lipid monogenic genes (Supplementary

320 Table 14). When accounting for both common and rare coding variants at the nearby familial hypercholesterolemia $L D L R$

321 gene, SPC24-enhancer DHS was significantly associated with total cholesterol $\left(p\right.$-value $\left.=3.01 \times 10^{-11}\right)$ and with suggestive

322 evidence for LDL-C (p-value $\left.=1.57 \times 10^{-06}\right)$. In a similarly adjusted model, LDLR-enhancer-DHS showed a strong

323 association with TC ( $\mathrm{p}$-value $\left.5.18 \times 10^{-12}\right)$. When adjusting for known common variants as well as rare coding variants in

324 PCSK9, both PCSK9-enhancer DHS and PCSK9-promoter DHS were significantly associated with total cholesterol. (Fig.

325 4, Supplementary Fig. 5). Through this procedure, CETP UTR retained significance for its independent association with

326 HDL-C as well as the putatively novel gene EHD3-promoter DHS association with TC. However, the non-coding gene-

327 centric $A P O C 3$ and $A P O E$ associations were rendered non-significant for HDL-C and TC, respectively.

328 Since we cannot rule out the possibility of reduced power for genome-wide rare variant analyses, we leveraged

329 current knowledge of 22 Mendelian lipid genes for more focused exploratory analyses ${ }^{14}$. We validated most genes in rare

330 variant coding analyses. The genes with the strongest coding signals typically had at least nominal evidence of gene-

331 centric non-coding rare variant associations (Supplementary Table 15, Supplementary Fig. 6). When rare coding 
332 variants were introduced into the model, the evidence for non-coding rare variant associations were largely unchanged.

333 Our findings expanding the currently described genetic basis for hypercholesterolemia to include rare non-coding variation

334 at LDLR and PCSK9 (Fig. 5).

\section{Discussion}

Conducting one of the largest population-based WGS association analyses, we now simultaneously interrogate

338 and establish a common, rare coding, and rare non-coding variant model for a complex trait. Utilizing 66,329 diverse

339 individuals with deep-coverage WGS, we interrogated 428M variants with plasma lipids expanding the allelic series to rare

340 non-coding variants, often within introns, of Mendelian lipid genes with prior robust rare coding variant support. Our

341 observations have important implications for plasma lipids as well as the genetic basis of complex traits more broadly.

342 WGS of diverse ancestries enables both allelic and locus heterogeneity for complex traits. Population genetic

343 analyses have largely been enriched for individuals of European descent ${ }^{37}$. Genetic association of plasma lipids using

344 arrays or whole exome sequencing among Europeans have yielded several important insights regarding plasma lipids and

345 the causal determinants of $\mathrm{CAD}^{5,4,38,39,40}$. Similar increasingly larger studies among non-Europeans have often yielded

346 new genetic loci and sometimes new genes, such as PCSK $9^{1,15,41,42,16}$. Such differences have also led to concerns about

347 the use of polygenic risk scores gleaned from much larger European GWAS of complex traits for non-Europeans ${ }^{43}$. Aided

348 by the availability of WGS data, we identify new putative loci associated with lipids in non-Europeans. Furthermore, our 
study enabled the discovery of several novel alleles at known loci, with richly distinct allelic heterogeneity across ancestry

350 groups. For example, HDL-C-raising CETP locus variants linked to CETP gene expression were only associated with

351 LDL-C reduction among those of African ancestry. While all pharmacologic CETP inhibitors increase HDL-C, only those

352 that decrease LDL-C also reduce cardiovascular disease risk ${ }^{27,28,30,29}$. Given the contribution of genetic differences,

353 clinical trials with more diverse samples would show insights.

354 Our study now provides increasingly robust evidence for a rare non-coding variant model for complex traits. Our

355 rare non-coding variant associations in both gene-centric and sliding window models were largely restricted to the introns

356 of Mendelian lipid genes with prior robust rare coding variant support consistent with biologic plausibility ${ }^{44}$. Rare intronic

357 variants, often impacting splicing, have been previously implicated in afflicted Mendelian families or small exceptional

358 case series, often through candidate gene approaches ${ }^{45,46,47,48}$. We discovered one example of a rare non-coding signal

359 without prior rare coding support - i.e., EHD3. We obtained estimates of phenotypic effect using burden tests. For most

360 regions, even nominal significance was not detected using burden testing indicating the likelihood of variants with

361 bidirectional effects further complicating clinical interpretation. When burden signals were detected, observed effects were

362 typically larger than common non-coding variants and less than rare coding variants, with the exception of $L D L R$,

363 consistent with whole genome mutational constraint models ${ }^{49,50,51}$.

364 The detection of independent rare non-coding variant signals has remained elusive largely due to limited sample

365 sizes with requisite WGS and limitations in the interpretation of rare non-coding variation functional consequence. 
Previously, we used annotated functional non-coding sequence in 16,324 TOPMed participants, and found that rare noncoding gene regions associated with lipid levels, but they were not independent of individually associated single variants $^{14}$. Using STAAR, we observed putative rare non-coding variant associations for lipids independent of individual variants associated with lipids in TOPMed.

WGS can improve diagnostic yield beyond the current standard of next-generation gene panel sequencing for dyslipidemias. A very small fraction with severe hypercholesterolemia and features consistent with strong genetic predisposition have a familial hypercholesterolemia variant in $L D L R, A P O B$, or $P C S K 9^{52,53}$. The presence of familial hypercholesterolemia variants is independently prognostic for CAD, beyond lipids, and merits the consideration of more costly lipid-lowering medications $s^{52,53,54,55}$. We now observe that rare $L D L R$ variants in Introns 2, 3, 16, and 17 lead to approximately 0.5 standard deviation increase in LDL-C, approximating effects observed with clinically reported exonic familial hypercholesterolemia variants in $L D L R^{55}$. Small studies have indicated the possibility of rare intronic $L D L R$ variants causing familial hypercholesterolemia due to altered splicing, which we now observe in our unbiased populationbased WGS study ${ }^{56,57}$. A WGS approach to lipid disorders, particularly for familial hypercholesterolemia, will markedly improve the diagnostic yield beyond existing limited approaches.

Our dynamic window approach may also improve the clinical curation of exonic variants. Among the data used to curate exonic variants is the use of in silico functional prediction tools ${ }^{58}$. Although evolutionary constraint measures are typically employed, such tools are largely agnostic to functional domain. As it relates to lipids, disruptive $A P O B$ and 
PCSK9 exonic variants can lead to strikingly opposing directions with large effects for LDL-C depending on

384 locations ${ }^{1,8,59,60}$. Using SCANG ${ }^{33}$, we detect a significant association with large effect for LDLR Exon 4 itself. This

385 observation supports the pathogenicity of $L D L R$ Exon 4 disruptive variants among patients with severe

386 hypercholesterolemia. The majority of familial hypercholesterolemia variants worldwide occur in Exon 4 of $L D L R^{61,62,63,64 .}$

387 Conventional rare coding variant analyses aggregate all exonic variants for a transcript. Here, we demonstrate an

388 opportunity for exon-level rare variant association testing.

389 Our study has important limitations. First, while our study is large for a WGS study by contemporary standards, it is

390 dwarfed by existing GWAS datasets limiting power for novel discovery. Nevertheless, by using WGS in diverse ancestries,

391 we can study hundreds of millions new variants. Second, prediction of rare non-coding variation consequence to prioritize

392 causal variants remains a challenge thereby limiting power ${ }^{65}$. The striking difference for most STAAR and burden results

393 also highlights bidirectional effects for rare non-coding variants within the same region and further challenges for clinical

394 utility. Third, given the paucity of multi-ancestral WGS datasets with lipids, our analyses are largely restricted to TOPMed.

395 For single variant associations, we pursued TOPMed-imputed GWAS datasets but were limited by the lack of ancestral

396 diversity. As TOPMed is a consortium of multiple different cohorts, we demonstrate consistencies by cohort. Furthermore,

397 rare variant non-coding signals were largely restricted to regions with rare variant coding signals supporting biological

398 plausibility. 
400 associated with lipids, including allelic heterogeneity at known loci and locus heterogeneity by ancestry. We characterize

401 the common, rare coding, and rare non-coding variant model for lipids. Lastly, we now demonstrate a monogenic-

402 equivalent model for rare $L D L R$ intronic variants predisposing to marked alterations in LDL-C, currently not recognized in

403 current population or clinical models for LDL-C.

404

405 Online Methods

406 Dataset

407 i) Contributing studies

408 The discovery cohort includes whole genome sequenced (WGS) data of 66,329 samples from 21 studies of the

409 Trans-Omics for Precision Medicine (TOPMed) program with blood lipids available ${ }^{17}$. The overall goal of TOPMed is to

410 generate and use trans-omics, including whole genome sequencing, of large numbers of individuals from diverse

411 ancestral backgrounds with rich phenotypic data to gain novel insights into heart, lung, blood, and sleep disorders. The

412 Freeze 8 data includes 140,306 samples out of which 66,329 samples qualified with lipid phenotype. Freeze 8 dataset

413 passed the central quality control protocol implemented by the TOPMed Informatics Research Core (described below)

414 and was deposited in the dbGaP TOPMed Exchange Area. 
The studies included in the current dataset, along with their abbreviations and sample sizes, contains the Old Order

416 Amish (Amish, $n=1,083$ ), Atherosclerosis Risk in Communities study (ARIC, $n=8,016)$, Mt Sinai BioMe Biobank (BioMe,

$417 \mathrm{n}=9,848)$, Coronary Artery Risk Development in Young Adults (CARDIA, $n=3,056)$, Cleveland Family Study (CFS, $\mathrm{n}=579$ ),

418 Cardiovascular Health Study (CHS, n=3,456), Diabetes Heart Study (DHS, n=365), Framingham Heart Study (FHS,

$419 \mathrm{n}=3,992)$, Genetic Studies of Atherosclerosis Risk (GeneSTAR, n=1,757), Genetic Epidemiology Network of Arteriopathy

420 (GENOA, n=1,046), Genetic Epidemiology Network of Salt Sensitivity (GenSalt, n=1,772), Genetics of Lipid-Lowering

421 Drugs and Diet Network (GOLDN, n=926), Hispanic Community Health Study - Study of Latinos (HCHS_SOL, n=7714),

422 Hypertension Genetic Epidemiology Network and Genetic Epidemiology Network of Arteriopathy (HyperGEN, n=1,853),

423 Jackson Heart Study (JHS, n=2,847), Multi-Ethnic Study of Atherosclerosis (MESA, $n=5,290$ ), Massachusetts General

424 Hospital Atrial Fibrillation Study (MGH_AF, n=683), San Antonio Family Study (SAFS, n=619), Samoan Adiposity Study

425 (SAS, $n=1,182$ ), Taiwan Study of Hypertension using Rare Variants (THRV, $n=1,982$ ) and Women's Health Initiative

426 (WHI, n=8,263) (Please see Supplementary Text for additional details). The multi-ancestral data set included individuals

427 from White (44\%), Black (26\%), Hispanic (21\%), Asian (7\%), and Samoan (2\%) ancestries. Study participants granted

428 consent per each study's Institutional Review Board (IRB) approved protocol. Secondarily, these data were analyzed

429 through a protocol approved by the Massachusetts General Hospital IRB. Supplementary Table 1 details the number of

430 samples across different studies and ancestral group. 
432 and Penn Medicine Biobanks which consist of 25,137 samples and 20,079 samples respectively ${ }^{26,25}$. We curated the

433 MGB Biobank and Penn Medicine Biobank phenotype data from the corresponding electronic health record databases in

434 accordance with corresponding institutional IRB approvals. Consent was previously obtained from each participant

435 regarding storage of biological specimens, genetic sequencing, access to all available electronic health record (EHR)

436 data, and permission to recontact for future studies. The MGB Biobank consists of 54\% and Penn Medicine Biobank

437 consist of $52 \%$ female samples and average ages were 55.89 years and 58.35 years, respectively (Supplementary

438 Table 5).

439

440 ii) Phenotypes

441 The primary outcomes in this study included LDL cholesterol (LDL-C), HDL cholesterol (HDL-C), total cholesterol

442 (TC) and triglycerides (TG) phenotypes. LDL-C was either directly measured or calculated by the Friedewald equation

443 when triglycerides were $<400 \mathrm{mg} / \mathrm{dL}$. Given the average effect of lipid lowering-medicines, when lipid-lowering medicines

444 were present, we adjusted the total cholesterol by dividing by 0.8 and LDL-C by dividing by 0.7 , as previously done ${ }^{14}$.

445 Triglycerides remained natural log transformed for analysis. Fasting status was accounted for with an indicator variable.

446 We harmonized the phenotypes across each cohort ${ }^{18}$ and inverse rank normalization of the residuals of each race

447 within each cohort scaled by the standard deviation of the trait and adjusted for covariates ${ }^{12}$. We included covariates such 
448 as age, age ${ }^{2}$, sex, PC1-11, study-groups as well as Mendelian founder lipid variants $A P O B$ p.R3527Q and $A P O C 3$

449 p.R19X for the Amish cohort ${ }^{7,66,8}$. Supplementary Table 1 provides the distributions of each of the four lipid phenotypes

450 by cohort, ancestral groups, and gender. We executed similar steps of phenotype harmonization and normalization for the

451 replication cohorts. Additionally, we adjusted the MGB Biobank for study-center and array-type, and Penn Medicine

452 Biobank for ancestry and BMI in addition to the other common covariates.

453

454 iii) Genotypes

455

Whole genome sequencing of goal $>30 X$ coverage was performed at seven centers (Broad Institute of MIT and

456 Harvard, Northwest Genomics Center, New York Genome Center, Illumina Genomic Services, PSOMAGEN [formerly

457 Macrogen], Baylor College of Medicine Human Genome Sequencing Center and McDonnell Genome Institute [MGI] at

458 Washington University). In most cases, all samples for a given study within a given Phase were sequenced at the same

459 center (Supplementary Text). The reads were aligned to human genome build GRCh38 using a common pipeline across

460 all centers (BWA-MEM).

461 The TOPMed Informatics Research Core at the University of Michigan performed joint genotype calling on all

462 samples in Freeze 8. The variant calling "GotCloud" pipeline (https://github.com/statgen/topmed_variant_calling) is under

463 continuous development and details on each step can be accessed through TOPMed website for Freeze8

464 (https://www.nhlbiwgs.org/topmed-whole-genome-sequencing-methods-freeze-8) ${ }^{17}$. The resulting BCF files were split by 
study and consent group for distribution to approved dbGaP users. Quality control was performed at each stage of the process, poor variant quality was indicated by missing rate $>20 \%$, mappability score $<0.8$, mean depth of coverage $>500 \mathrm{X}$, and Ti/Tv ratio, by the Sequencing Centers, the IRC and the TOPMed Data Coordinating Center (DCC). The VCF/BCF files were converted to GDS (Genomic Data Structure) format by the DCC and were deposited into the dbGap TOPMed Exchange Area.

The genetic relationship matrix (GRM) is an $N^{*} \mathrm{~N}$ matrix of relatedness information of the samples included in the study and was computed centrally using 'PC-relate' R package (version: 1.24.0) ${ }^{67}$. Using the 'Genesis' R package (version:2.20.1 ${ }^{68}$ we generated subsetted GRM for the samples with plasma lipid profiles. The GDS files with the variants were annotated internally by curating data from multiple database sources using Functional Annotation of Variant-Online Resource (FAVOR (http://favor.genohub.org) ${ }^{13}$. This study used the resultant aGDS (annotation GDS) files.

The MGB Biobank replication cohort was genotyped using three different arrays (Multiethnic Exome Global (Meg), Human multi-ethnic array (Mega), and Expanded multi-ethnic genotyping array (Megex)), and we separately imputed the data using TOPMed imputation server with default parameters ${ }^{69,70}$. This study applied the Version-r2 of the imputation panel, it includes 97,256 reference samples and $\sim 300 \mathrm{M}$ genetic variants. The Illumina Global Screening array was used to genotype the Penn Medicine Biobank. Penn Medicine Biobank TOPMed imputation was performed using EAGLE ${ }^{70}$ and Minimac ${ }^{71}$ software. For this study we downloaded variants that passed a min $\mathrm{R}^{2}$ threshold of 0.3 . 


97

\section{Single Variant Association}

We performed genome-wide single variant association analyses for autosomal variants with minor allele frequency (MAF) greater than $0.1 \%$ across the dataset with each of the four lipid phenotypes. We implemented the SAIGE-QT ${ }^{72}$ method, which employs fast linear mixed models with kinship adjustment, in Encore (https://encore.sph.umich.edu/) for single variant association analyses. We additionally adjusted the model for covariates (PC1-PC11, age, sex, age ${ }^{2}$, and study-groups [cohort-race subgrouping]).

We conducted single variant association replications for putative novel variants. After comparing the results with published lipid GWAS summary statistics, we filtered putative novel GWAS variants based on a stringent whole genomewide significant threshold (alpha $\left.=5 \times 10^{-9}\right)^{73}$. Replication was performed in the MGB and Penn Medicine Biobanks where models were fitted as indicated above. Additionally, we adjusted the MGB Biobank for study recruitment center and array and Penn Medicine Biobank for ancestry and BMI. In the MGB Biobank, we selected lipid concentrations closest to the sample acquisition time point and adjusted for statins if prescribed within one year prior to sample acquisition. In the Penn

\section{Biobank, we utilized each participant's median lipid concentration for replication; statins prescribed prior to lipid} concentration used were adjusted in the models. Additionally, we carried out meta-analysis using fixed effects model based on inverse-variance-weighted effect size for the two replication cohorts using METASOFT ${ }^{74}$.

\section{Rare variant association test}


500 infoRmation (STAAR) pipeline ${ }^{13}$ from STARtopmed R package. STAARpipeline is a regression-based framework that

501 permits adjustment of covariates, population structure, and relatedness by fitting linear and logistic mixed models for

502 quantitative and dichotomous traits ${ }^{75}$. We chose STAAR to leverage the annotation information and associated scores

503 that were available for TOPMed Freeze 8 data to incorporate the analysis of rare non-coding variants from whole genome

504 sequencing. The method implements genome-wide scanning of rare variants (MAF<0.01) in gene-centric and region-

505 based workflows. For each variant set, STAARpipeline calculates a set-based p-value using the STAAR method, which

506 increases the analysis power by incorporating multiple in silico variant functional annotation scores capturing diverse

507 genomic features and biochemical readouts ${ }^{13}$. We aggregated rare variants into multiple groups for coding and non-

508 coding analyses. For the coding region, we defined five different aggregate masks of rare variants 1) plof (putative loss-of-

509 function), plof-Ds (putative loss-of-function or disruptive missense), missense, disruptive-missense, and synonymous. For

510 the non-coding regions, we used seven rare variant masks: 1) promoter-CAGE (promoter variants within Cap Analysis of

511 Gene Expression [CAGE] sites ${ }^{77,78}$ ), 2) promoter-DHS (promoter variants within DNase hypersensitivity [DHS] sites ${ }^{79}$ ), 3)

512 enhancer-CAGE (enhancer within CAGE sites ${ }^{78}$ ), 4) enhancer-DHS (enhancer variants within DHS sites ${ }^{80}$ ), 5) UTR (rare

513 variants in 3' untranslated region [UTR] and 5' UTR untranslated region), 6) upstream, and 7) downstream. Detailed

514 explanations of the regions defined based on these masks is discussed within STAARpipeline ${ }^{13}$. 
516 window of transcription starting site (TSS), enhancer: GeneHancer predicted regions) regions, we considered only genes

517 with at least two rare variants (i.e., 18,445 genes in all 22 autosomes). In the region-based workflows, we implemented two

518 protocols: 1) a 'sliding window' approach, where we aggregated rare variants within 2-kb sliding windows and with 1-kb

519 overlap length, and 2) a 'dynamic window' approach, where we executed SCANG ${ }^{33}$ method and aggregated dynamically

520 variant-sets between 40-300 variants per set, where the method systematically scans the whole genome with overlapping

521 windows of varying sizes. The STAARtopmed R-package implements multiple rare-variants aggregate tests including

$522 \mathrm{SKAT}^{81}$, Burden $^{82}$ and $\mathrm{ACAT}^{83}$ and integrates them as STAAR-O ${ }^{13}$. We performed gene-centric and region-based rare

523 variant tests using annotated GDS files of TOPMed.

$524 \quad$ We completed aggregate tests as three-step process. In the first step, we fitted a null model using glmmkin()

525 function in STAARtopmed. The null model was fitted for each of the four lipid phenotypes adjusted for all covariates and

526 relatedness except the genotype of interest. In the second step, we ran genome wide gene-centric and region-based rare-

527 variant aggregate tests. The third step directed conditional analyses, where the results were adjusted for previously

528 known significantly lipid-associated (i.e., $p<5 \times 10^{-8}$ in external datasets) individual variants from GWAS Catalog ${ }^{84}$ and

529 Million Veterans Program (MVP) ${ }^{15}$ GWAS summary statistics. To obtain effect estimates of significant aggregate sets, we

530 associated the cumulative genotypes (binary scores) based on the variants forming the aggregates and used Glmm.Wald 
531 test from GMMAT R package ${ }^{75}$ (version 1.3.1). For significantly-associated window-based rare variant aggregations, we

532 trimmed the exonic variants and estimated the effects with only non-coding variants.

\section{CETP gene expression and lipid trait colocalization}

535 We studied the correlation of LDL-C and HDL-C effects with eQTL effects at chromosome 16q13, which includes

536 CETP. We downloaded GTEx eQTL build 38 (version8) data for Liver, Adipose Subcutaneous and Adipose Visceral

537 (Omentum) tissues from GTEx Portal on 16/APR/2020 ${ }^{85}$. We selected eQTLs with nominal significance ( $p$-value<0.05)

538 and utilized the eQTL-gene pairs with the most significant p-values. Genes with at least 5 eQTLs were selected for the

539 colocalization analysis. We selected variants with a suggestive significance ( $p$-value $<5 \times 10^{-7}$ ) for LDL-C or HDL-C effects

540 within $500 \mathrm{~kb}$ of the lead locus variant. We performed Pearson correlation tests between the lipid effect estimates and

541 gene expression effects (slope) from GTEX.

542 
544 Whole genome sequencing (WGS) for the Trans-Omics in Precision Medicine (TOPMed) program was supported by the

545 National Heart, Lung and Blood Institute (NHLBI). P.N. is supported by grants from the National Heart, Lung, and Blood

546 Institute (R01HL142711, R01HL148050, R01HL151283, R01HL148565, R01HL135242, R01HL151152), Fondation

547 Leducq (TNE-18CVD04), and Massachusetts General Hospital (Paul and Phyllis Fireman Endowed Chair in Vascular

548 Medicine). The Amish studies were supported by NIH grants R01 AG18728, U01 HL072515, R01 HL088119, R01

549 HL121007, and P30 DK072488. The Atherosclerosis Risk in Communities (ARIC) study has been funded in whole or in

550 part with Federal funds from the National Heart, Lung, and Blood Institute, National Institutes of Health, Department of

551 Health and Human Services (contract numbers HHSN268201700001I, HHSN268201700002I, HHSN268201700003I,

552 HHSN268201700004I and HHSN268201700005I). The authors thank the staff and participants of the ARIC study for their

553 important contributions. The Mount Sinai BioMe Biobank (BioMe) has been supported by The Andrea and Charles

554 Bronfman Philanthropies and in part by Federal funds from the NHLBI and NHGRI (U01HG00638001; U01HG007417;

555 X01HL134588). Coronary Artery Risk Development in Young Adults (CARDIA) Study (phs001612) was performed at the

556 Baylor College of Medicine Human genome Sequencing Center (contract HHSN268201600033I). Core support including

557 centralized genomic read mapping and genotype calling, along with variant quality metrics and filtering were provided by

558 the TOPMed Informatics Research Center (3R01HL-117626-02S1; contract HHSN268201800002I). Core support

559 including phenotype harmonization, data management, sample-identity QC, and general program coordination were 
560 provided by the TOPMed Data Coordinating Center (R01HL-120393; U01HL-120393; contract HHSN268201800001I).

561 We gratefully acknowledge the studies and participants who provided biological samples and data for TOPMed. The

562 Coronary Artery Risk Development in Young Adults Study (CARDIA) is conducted and supported by the National Heart,

563 Lung, and Blood Institute (NHLBI) in collaboration with the University of Alabama at Birmingham (HHSN268201800005I \&

564 HHSN268201800007I), Northwestern University (HHSN268201800003I), University of Minnesota (HHSN268201800006I),

565 and Kaiser Foundation Research Institute (HHSN268201800004I). Cleveland Family Study (CFS) is supported by grants

566 from the NHLBI (HL046389, HL113338, and 1R35HL135818). Cardiovascular Health Study (CHS) was supported by

567 contracts HHSN268201200036C, HHSN268200800007C, HHSN268201800001C, N01HC55222, N01HC85079,

568 N01HC85080, N01HC85081, N01HC85082, N01HC85083, N01HC85086, 75N92021D00006, and grants U01HL080295

569 and U01HL130114 from the National Heart, Lung, and Blood Institute (NHLBI), with additional contribution from the

570 National Institute of Neurological Disorders and Stroke (NINDS). Additional support was provided by R01AG023629 from

571 the National Institute on Aging (NIA). A full list of principal CHS investigators and institutions can be found at CHS-

572 NHLBI.org. The content is solely the responsibility of the authors and does not necessarily represent the official views of

573 the National Institutes of Health. Diabetes Heart Study (DHS) was supported by HL92301, HL67348, NS058700,

574 AR48797, DK071891, AG058921, the General Clinical Research Center of the Wake Forest University School of

575 Medicine (RR07122, HL085989), the American Diabetes Association, and a pilot grant from the Claude Pepper Older

576 Americans Independence Center of Wake Forest University Health Sciences (AG10484). Framingham Heart Study (FHS) 
577 acknowledges the support of contracts NO1-HC-25195 and HHSN268201500001I from the National Heart, Lung and

578 Blood Institute and grant supplement R01 HL092577-06S1 for this research. WGS for "NHLBI TOPMed: Whole Genome

579 Sequencing and Related Phenotypes in the Framingham Heart Study" (phs000974) was performed at the Broad Institute

580 of MIT and Harvard (HHSN268201500014C, 3R01HL092577-06S1, and 3U54HG003067-12S2). We also acknowledge

581 the dedication of the FHS study participants without whom this research would not be possible. Genetic Studies of

582 Atherosclerosis Risk (GeneSTAR) was supported by grants from the National Institutes of Health/National Heart, Lung,

583 and Blood Institute (U01 HL72518, HL087698, HL49762, HL58625, HL071025, HL112064), the National Institutes of

584 Health/National Institute of Nursing Research (NR0224103), and by a grant from the National Institutes of Health/National

585 Center for Research Resources (M01-RR000052) to the Johns Hopkins General Clinical Research Center. Genetic

586 Epidemiology Network of Arteriopathy (GENOA) was supported by the National Heart, Lung and Blood Institute

587 (HL054457, HL054464, HL054481, HL087660, and HL119443) of the National Institutes of Health. Genetic Epidemiology

588 Network of Salt Sensitivity (GenSalt) was supported by research grants (U01HL072507, R01HL087263, and

589 R01HL090682) from the National Heart, Lung and Blood Institute, National Institutes of Health, Bethesda, MD. Genetics

590 of Lipid-Lowering Drugs and Diet Network (GOLDN) biospecimens, baseline phenotype data, and intervention phenotype

591 data were collected with funding from National Heart, Lung and Blood Institute (NHLBI) grant U01 HL072524. The

592 Hispanic Community Health Study/Study of Latinos (HCHS-SOL) was carried out as a collaborative study supported by

593 contracts from the National Heart, Lung, and Blood Institute (NHLBI) to the University of North Carolina (N01-HC65233), 
594 University of Miami (N01-HC65234), Albert Einstein College of Medicine (N01-HC65235), Northwestern University (N01-

595 HC65236), and San Diego State University (N01-HC65237). The Hypertension Genetic Epidemiology Network and

596 Genetic Epidemiology Network of Arteriopathy (HyperGEN) Study is part of the National Heart, Lung, and Blood Institute

597 (NHLBI) Family Blood Pressure Program; collection of the data represented here was supported by grants U01 HL054472

598 (MN Lab), U01 HL054473 (DCC), U01 HL054495 (AL FC), and U01 HL054509 (NC FC). The HyperGEN: Genetics of Left

599 Ventricular Hypertrophy Study was supported by NHLBI grant R01 HL055673 with whole-genome sequencing made

600 possible by supplement $-18 \mathrm{~S} 1$. The Jackson Heart Study (JHS) is supported and conducted in collaboration with Jackson

601 State University (HHSN268201800013I), Tougaloo College (HHSN268201800014I), the Mississippi State

602 Department of Health (HHSN268201800015I) and the University of Mississippi Medical Center

603 (HHSN268201800010I, HHSN268201800011I and HHSN268201800012I) contracts from the National Heart, Lung,

604 and Blood Institute (NHLBI) and the National Institute on Minority Health and Health Disparities (NIMHD). The

605 authors also wish to thank the staffs and participants of the JHS. Multi-Ethnic Study of Atherosclerosis (MESA) and the

606 MESA SHARe projects are conducted and supported by the National Heart, Lung, and Blood Institute (NHLBI) in

607 collaboration with MESA investigators. Support for MESA is provided by contracts 75N92020D00001,

608 HHSN268201500003I, N01-HC-95159, 75N92020D00005, N01-HC-95160, 75N92020D00002, N01-HC-95161,

609 75N92020D00003, N01-HC-95162, 75N92020D00006, N01-HC-95163, 75N92020D00004, N01-HC-95164,

610 75N92020D00007, N01-HC-95165, N01-HC-95166, N01-HC-95167, N01-HC-95168, N01-HC-95169, UL1-TR-000040, 
611 UL1-TR-001079, and UL1-TR-001420. Funding for SHARe genotyping was provided by NHLBI Contract N02-HL-64278.

612 Genotyping was performed at Affymetrix (Santa Clara, California, USA) and the Broad Institute of Harvard and MIT

613 (Boston, Massachusetts, USA) using the Affymetrix Genome-Wide Human SNP Array 6.0. Also supported in part by

614 NHLBI CHARGE Consortium Contract HL105756. The provision of genotyping data was supported in part by the National

615 Center for Advancing Translational Sciences, CTSI grant UL1TR001881, and the National Institute of Diabetes and

616 Digestive and Kidney Disease Diabetes Research Center (DRC) grant DK063491 to the Southern California Diabetes

617 Endocrinology Research Center. Infrastructure for the CHARGE Consortium is supported in part by the National Heart,

618 Lung, and Blood Institute (NHLBI) grant R01HL105756. The Massachusetts General Hospital Atrial Fibrillation Study

619 (MGH-AF) was supported by grants to Dr. Ellinor from the Fondation Leducq (14CVD01), the National Institutes of Health

620 to Dr. Ellinor (1RO1HL092577, R01HL128914, K24HL105780) and Dr. Lubitz (1R01HL139731) and by grants from the

621 American Heart Association to Dr. Ellinor (18SFRN34110082) and to Dr. Lubitz (18SFRN34250007). San Antonio Family

622 Study (SAFS) was supported in part by National Institutes of Health (NIH) grants R01 HL045522, MH078143, MH078111

623 and MH083824; and whole genome sequencing of SAFS subjects was supported by U01 DK085524 and R01 HL113323.

624 We are very grateful to the participants of the San Antonio Family Study for their continued involvement in our research

625 programs. Samoan Adiposity Study (SAS) was funded by NIH grant R01-HL093093. We thank the Samoan participants of

626 the study and local village authorities. We acknowledge the support of the Samoan Ministry of Health and the Samoa

627 Bureau of Statistics for their support of this research. The Rare Variants for Hypertension in Taiwan Chinese (THRV) is 
628 supported by the National Heart, Lung, and Blood Institute (NHLBI) grant (R01HL111249) and its participation in TOPMed

629 is supported by an NHLBI supplement (R01HL111249-04S1). SAPPHIRe was supported by NHLBI grants (U01HL54527,

630 U01HL54498) and Taiwan funds, and the other cohorts were supported by Taiwan funds. The Women's Health Initiative

631 (WHI) program is funded by the National Heart, Lung, and Blood Institute, National Institutes of Health, U.S. Department

632 of Health and Human Services through contracts HHSN268201600018C, HHSN268201600001C, HHSN268201600002C,

633 HHSN268201600003C, and HHSN268201600004C. The Centers for Common Disease Genomics (CCDG) program was

634 supported by NHGRI and NHLBI, and whole genome sequencing was performed at the Baylor College of Medicine

635 Human Genome Sequencing Center (UM1 HG008898 and R01HL059367). We like to acknowledge all the grants that

636 supported this study, R01 HL121007, U01 HL072515, R01 AG18728, X01HL134588, HL 046389, HL113338, and

637 1R35HL135818, K01 HL135405, R03 HL154284, U01HL072507, R01HL087263, R01HL090682, P01HL045522,

638 R01MH078143, R01MH078111, R01MH083824, U01DK085524, R01HL113323, R01HL093093, R01HL140570,

639 R01HL142711, R01HL127564, R01HL148050, R01HL148565, and Leducq TNE-18CVD04. The views expressed in this

640 manuscript are those of the authors and do not necessarily represent the views of the National Heart, Lung, andBlood

641 Institute; the National Institutes of Health; or the U.S.Department of Health and Human Services.

642

643 Author's information

644 NHLBI Trans-Omics for Precision Medicine (TOPMed) Consortium 
645 Namiko Abe ${ }^{63}$, Gonçalo Abecasis ${ }^{64}$, Francois Aguet ${ }^{65}$, Christine Albert $^{66}$, Laura Almasy ${ }^{67}$, Alvaro Alonso ${ }^{68}$, Seth Ament ${ }^{69}$, 646 Peter Anderson ${ }^{70}$, Pramod Anugu ${ }^{71}$, Deborah Applebaum-Bowden ${ }^{72}$, Kristin Ardlie ${ }^{65}$, Dan Arking ${ }^{73}$, Allison Ashley-Koch ${ }^{74}$,

647 Tim Assimes $^{75}$, Paul Auer ${ }^{76}$, Dimitrios Avramopoulos ${ }^{73}$, Najib Ayas ${ }^{77}$, Adithya Balasubramanian ${ }^{78}$, John Barnard ${ }^{79}$,

648 Kathleen Barnes ${ }^{80}$, R. Graham Barr ${ }^{81}$, Emily Barron-Casella ${ }^{73}$, Lucas Barwick ${ }^{82}$, Terri Beaty ${ }^{73}$, Gerald Beck ${ }^{83}$, Diane

649 Becker $^{84}$, Lewis Becker ${ }^{73}$, Rebecca Beer ${ }^{85}$, Amber Beitelshees $^{69}$, Emelia Benjamin ${ }^{86}$, Takis Benos ${ }^{87}$, Marcos Bezerra ${ }^{88}$,

650 Larry Bielak $^{64}$, Thomas Blackwell ${ }^{64}$, Russell Bowler ${ }^{89}$, Ulrich Broeckel ${ }^{90}$, Jai Broome ${ }^{70}$, Deborah Brown ${ }^{91}$, Karen Bunting ${ }^{63}$,

651 Esteban Burchard $^{92}$, Carlos Bustamante ${ }^{93}$, Erin Buth ${ }^{94}$, Jonathan Cardwell ${ }^{95}$, Vincent Carey ${ }^{96}$, Julie Carrier ${ }^{97}$, Cara

652 Carty $^{98}$, Richard Casaburi ${ }^{99}$, Juan P Casas Romero ${ }^{100}$, James Casella ${ }^{73}$, Peter Castaldi ${ }^{101}$, Mark Chaffin ${ }^{65}$, Christy

653 Chang $^{69}$, Yi-Cheng Chang ${ }^{102}$, Daniel Chasman ${ }^{103}$, Sameer Chavan ${ }^{95}$, Bo-Juen Chen ${ }^{63}$, Wei-Min Chen ${ }^{104}$, Yii-Der Ida

654 Chen $^{105}$, Michael Cho ${ }^{96}$, Seung Hoan Choi ${ }^{65}$, Mina Chung ${ }^{106}$, Clary Clish ${ }^{107}$, Suzy Comhair ${ }^{108}$, Matthew Conomos ${ }^{94}$,

655 Elaine Cornell ${ }^{109}$, Carolyn Crandall ${ }^{99}$, James Crapo ${ }^{110}$, L. Adrienne Cupples ${ }^{111}$, Jeffrey Curtis ${ }^{64}$, Brian Custer ${ }^{112}$, Coleen

656 Damcott $^{69}$, Dawood Darbar ${ }^{113}$, Sean David ${ }^{114}$, Colleen Davis ${ }^{70}$, Michelle Daya ${ }^{95}$, Mariza de Andrade ${ }^{115}$, Michael

657 DeBaun ${ }^{116}$, Ranjan Deka ${ }^{117}$, Dawn DeMeo ${ }^{96}$, Scott Devine ${ }^{69}$, Huyen Dinh ${ }^{78}$, Harsha Doddapaneni ${ }^{78}$, Qing Duan ${ }^{118}$,

658 Shannon Dugan-Perez ${ }^{78}$, Ravi Duggirala ${ }^{119}$, Jon Peter Durda ${ }^{109}$, Charles Eaton ${ }^{120}$, Lynette Ekunwe ${ }^{71}$, Adel El Boueiz ${ }^{121}$,

659 Leslie Emery $^{70}$, Serpil Erzurum ${ }^{79}$, Charles Farber ${ }^{104}$, Jesse Farek ${ }^{78}$, Tasha Fingerlin ${ }^{122}$, Matthew Flickinger ${ }^{64}$, Nora

660 Franceschini $^{123}$, Chris Frazar ${ }^{70}$, Mao Fu ${ }^{69}$, Stephanie M. Fullerton ${ }^{70}$, Lucinda Fulton ${ }^{124}$, Weiniu Gan ${ }^{85}$, Shanshan Gao ${ }^{95}$,

661 Yan Gao ${ }^{71}$, Margery Gass ${ }^{125}$, Heather Geiger ${ }^{126}$, Bruce Gelb ${ }^{127}$, Mark Geraci ${ }^{128}$, Robert Gerszten ${ }^{129}$, Auyon Ghosh ${ }^{96}$, 
662 Chris Gignoux ${ }^{75}$, Mark Gladwin ${ }^{87}$, David Glahn ${ }^{130}$, Stephanie Gogarten ${ }^{70}$, Da-Wei Gong ${ }^{69}$, Harald Goring ${ }^{131}$, Sharon 663 Graw $^{80}$, Kathryn J. Gray ${ }^{132}$, Daniel Grine ${ }^{95}$, Colin Gross ${ }^{64}$, C. Charles Gu ${ }^{124}$, Yue Guan ${ }^{69}$, Namrata Gupta ${ }^{65}$, David M.

664 Haas $^{133}$, Jeff Haessler ${ }^{125}$, Michael Hall ${ }^{134}$, Yi Han $^{78}$, Patrick Hanly ${ }^{135}$, Daniel Harris ${ }^{136}$, Nicola L. Hawley ${ }^{137}$, Ben Heavner ${ }^{94}$, 665 Susan Heckbert ${ }^{138}$, Ryan Hernandez ${ }^{92}$, David Herrington ${ }^{139}$, Craig Hersh ${ }^{140}$, Bertha Hidalgo ${ }^{141}$, James Hixson ${ }^{142}$, Brian 666 Hobbs $^{96}$, John Hokanson ${ }^{95}$, Elliott Hong ${ }^{69}$, Karin Hoth ${ }^{143}$, Chao (Agnes) Hsiung ${ }^{144}$, Jianhong Hu ${ }^{78}$, Yi-Jen Hung ${ }^{145}$, Haley

667 Huston $^{146}$, Chii Min Hwu ${ }^{147}$, Rebecca Jackson ${ }^{148}$, Deepti Jain ${ }^{70}$, Cashell Jaquish ${ }^{85}$, Jill Johnsen ${ }^{149}$, Andrew Johnson ${ }^{85}$, 668 Craig Johnson ${ }^{70}$, Rich Johnston ${ }^{68}$, Kimberly Jones ${ }^{73}$, Hyun Min Kang ${ }^{150}$, Shannon Kelly ${ }^{151}$, Eimear Kenny ${ }^{127}$, Michael

669 Kessler $^{69}$, Alyna Khan ${ }^{70}$, Ziad Khan ${ }^{78}$, Wonji Kim ${ }^{152}$, John Kimoff ${ }^{153}$, Greg Kinney ${ }^{154}$, Barbara Konkle ${ }^{146}$, Holly Kramer ${ }^{155}$, 670 Christoph Lange ${ }^{156}$, Ethan Lange ${ }^{95}$, Cathy Laurie ${ }^{70}$, Cecelia Laurie ${ }^{70}$, Meryl LeBoff ${ }^{96}$, Jiwon Lee ${ }^{96}$, Sandra Lee ${ }^{78}$, Wen-

671 Jane Lee ${ }^{147}$, Jonathon LeFaive ${ }^{64}$, David Levine ${ }^{70}$, Dan Levy ${ }^{85}$, Joshua Lewis ${ }^{69}$, Yun Li ${ }^{118}$, Henry Lin ${ }^{105}$, Honghuang Lin ${ }^{157}$,

672 Simin Liu ${ }^{158}$, Yongmei Liu ${ }^{159}$, Yu Liu ${ }^{160}$, Kathryn Lunetta ${ }^{157}$, James Luo ${ }^{85}$, Ulysses Magalang ${ }^{161}$, Michael Mahaney ${ }^{162}$, 673 Barry Make ${ }^{73}$, Alisa Manning ${ }^{163}$, JoAnn Manson ${ }^{96}$, Lisa Martin ${ }^{164}$, Melissa Marton ${ }^{126}$, Susan Mathai ${ }^{95}$, Susanne May ${ }^{94}$, 674 Patrick McArdle ${ }^{69}$, Merry-Lynn McDonald ${ }^{141}$, Sean McFarland ${ }^{152}$, Daniel McGoldrick ${ }^{165}$, Caitlin McHugh ${ }^{94}$, Becky $675 \mathrm{McNeil}^{166}$, Hao Mei ${ }^{71}$, James Meigs ${ }^{167}$, Vipin Menon ${ }^{78}$, Luisa Mestroni ${ }^{80}$, Ginger Metcalf ${ }^{78}$, Deborah A Meyers ${ }^{168}$, 676 Emmanuel Mignot ${ }^{169}$, Julie Mikulla ${ }^{85}$, Nancy Min ${ }^{71}$, Mollie Minear ${ }^{170}$, Ryan L Minster ${ }^{87}$, Matt Moll ${ }^{101}$, Zeineen Momin ${ }^{78}$, 677 Courtney Montgomery ${ }^{171}$, Donna Muzny ${ }^{78}$, Josyf C Mychaleckyj ${ }^{104}$, Girish Nadkarni ${ }^{127}$, Rakhi Naik ${ }^{73}$, Sergei Nekhai ${ }^{172}$, 678 Sarah C. Nelson ${ }^{94}$, Bonnie Neltner ${ }^{95}$, Caitlin Nessner ${ }^{78}$, Osuji Nkechinyere ${ }^{78}$, Jeff O'Connell ${ }^{173}$, Tim O'Connor ${ }^{69}$, Heather 
679 Ochs-Balcom ${ }^{174}$, Geoffrey Okwuonu ${ }^{78}$, Allan Pack ${ }^{175}$, David T. Paik ${ }^{176}$, James Pankow ${ }^{177}$, George Papanicolaou ${ }^{85}$, Cora

680 Parker $^{178}$, Juan Manuel Peralta ${ }^{119}$, Marco Perez ${ }^{75}$, James Perry ${ }^{69}$, Ulrike Peters ${ }^{179}$, Lawrence S Phillips ${ }^{68}$, Jacob

681 Pleiness $^{64}$, Toni Pollin ${ }^{69}$, Wendy Post ${ }^{180}$, Julia Powers Becker ${ }^{181}$, Meher Preethi Boorgula ${ }^{95}$, Michael Preuss ${ }^{127}$, Pankaj

682 Qasba $^{85}$, Dandi Qiao ${ }^{96}$, Zhaohui Qin ${ }^{68}$, Nicholas Rafaels ${ }^{182}$, Laura Raffield ${ }^{183}$, Mahitha Rajendran ${ }^{78}$, Vasan S.

683 Ramachandran ${ }^{157}$, D.C. Rao ${ }^{124}$, Laura Rasmussen-Torvik ${ }^{184}$, Aakrosh Ratan ${ }^{104}$, Robert Reed ${ }^{69}$, Catherine Reeves ${ }^{185}$,

684 Elizabeth Regan ${ }^{110}$, Alex Reiner ${ }^{186}$, Muagututi'a Sefuiva Reupena ${ }^{187}$, Ken Rice ${ }^{70}$, Rebecca Robillard ${ }^{188}$, Nicolas Robine ${ }^{126}$,

685 Dan Roden ${ }^{189}$, Carolina Rosellii ${ }^{65}$, Ingo Ruczinski ${ }^{73}$, Alexi Runnels ${ }^{126}$, Pamela Russell ${ }^{95}$, Sarah Ruuska ${ }^{146}$, Kathleen

686 Ryan $^{69}$, Ester Cerdeira Sabino ${ }^{190}$, Danish Saleheen ${ }^{191}$, Shabnam Salimi ${ }^{69}$, Sejal Salvi ${ }^{78}$, Steven Salzberg ${ }^{73}$, Kevin

687 Sandow $^{192}$, Vijay G. Sankaran ${ }^{193}$, Jireh Santibanez ${ }^{78}$, Karen Schwander ${ }^{124}$, David Schwartz ${ }^{95}$, Frank Sciurba ${ }^{87}$, Christine

688 Seidman ${ }^{194}$, Jonathan Seidman ${ }^{195}$, Frédéric Sériès ${ }^{196}$, Vivien Sheehan ${ }^{197}$, Stephanie L. Sherman ${ }^{198}$, Amol Shetty $^{69}$, Aniket

689 Shetty ${ }^{95}$, Wayne Hui-Heng Sheu ${ }^{147}$, M. Benjamin Shoemaker ${ }^{199}$, Brian Silver ${ }^{200}$, Edwin Silverman ${ }^{96}$, Robert Skomro ${ }^{201}$,

690 Albert Vernon Smith ${ }^{202}$, Josh Smith ${ }^{70}$, Nicholas Smith ${ }^{138}$, Tanja Smith ${ }^{63}$, Sylvia Smoller ${ }^{203}$, Beverly Snively ${ }^{204}$, Michael

691 Snyder $^{75}$, Tamar Sofer $^{96}$, Nona Sotoodehnia ${ }^{70}$, Adrienne M. Stilp ${ }^{70}$, Garrett Storm ${ }^{205}$, Elizabeth Streeten ${ }^{69}$, Jessica Lasky

$692 \mathrm{Su}^{96}$, Yun Ju Sung ${ }^{124}$, Jody Sylvia ${ }^{96}$, Adam Szpiro ${ }^{70}$, Daniel Taliun ${ }^{64}$, Hua Tang ${ }^{206}$, Margaret Taub ${ }^{73}$, Matthew Taylor ${ }^{80}$,

693 Simeon Taylor ${ }^{69}$, Marilyn Telen ${ }^{74}$, Timothy A. Thornton ${ }^{70}$, Machiko Threlkeld ${ }^{207}$, Lesley Tinker ${ }^{125}$, David Tirschwell ${ }^{70}$,

694 Sarah Tishkoff ${ }^{208}$, Hemant Tiwari ${ }^{209}$, Catherine Tong $^{210}$, Dhananjay Vaidya ${ }^{73}$, David Van Den Berg ${ }^{211}$, Peter VandeHaar ${ }^{64}$,

695 Scott Vrieze ${ }^{177}$, Tarik Walker $^{95}$, Robert Wallace ${ }^{143}$, Avram Walts $^{95}$, Fei Fei Wang ${ }^{70}$, Heming Wang ${ }^{212}$, Jiongming Wang ${ }^{202}$, 
696 Karol Watson $^{99}$, Jennifer Watt ${ }^{78}$, Daniel E. Weeks ${ }^{87}$, Joshua Weinstock ${ }^{150}$, Bruce Weir ${ }^{70}$, Scott T Weiss ${ }^{213}$, Lu-Chen

697 Weng $^{214}$, Jennifer Wessel ${ }^{215}$, Kayleen Williams ${ }^{94}$, L. Keoki Williams ${ }^{216}$, Carla Wilson ${ }^{96}$, James Wilson ${ }^{217}$, Lara

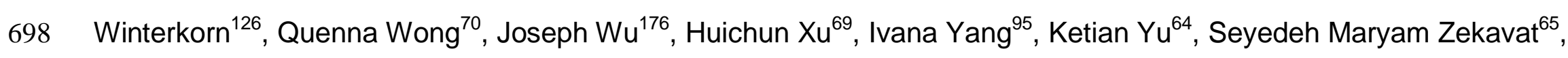

699 Yingze Zhang ${ }^{218}$, Snow Xueyan Zhao ${ }^{110}$, Wei Zhao ${ }^{219}$, Xiaofeng Zhu ${ }^{220}$, Michael Zody ${ }^{63}$, Sebastian Zoellner ${ }^{64}$

700

70163 - New York Genome Center, New York, New York, 10013; 64 - University of Michigan, Ann Arbor, Michigan, $48109 ; 65$

702 - Broad Institute, Cambridge, Massachusetts, 02142; 66 - Cedars Sinai, Boston, Massachusetts, 02114; 67 - Children's

703 Hospital of Philadelphia, University of Pennsylvania, Philadelphia, Pennsylvania, 19104; 68 - Emory University, Atlanta,

704 Georgia, 30322; 69 - University of Maryland, Baltimore, Maryland, 21201; 70 - University of Washington, Seattle,

705 Washington, 98195; 71 - University of Mississippi, Jackson, Mississippi, 38677; 72 - National Institutes of Health,

706 Bethesda, Maryland, 20892; 73 - Johns Hopkins University, Baltimore, Maryland, 21218; 74 - Duke University, Durham,

707 North Carolina, 27708; 75 - Stanford University, Stanford, California, 94305; 76 - University of Wisconsin Milwaukee,

708 Milwaukee, Wisconsin, 53211; 77 - Providence Health Care, Medicine, Vancouver; 78 - Baylor College of Medicine

709 Human Genome Sequencing Center, Houston, Texas, 77030; 79 - Cleveland Clinic, Cleveland, Ohio, $44195 ; 80$ -

710 University of Colorado Anschutz Medical Campus, Aurora, Colorado, 80045; 81 - Columbia University, New York, New

711 York, 10032; 82 - The Emmes Corporation, LTRC, Rockville, Maryland, 20850; 83 - Cleveland Clinic, Quantitative Health

712 Sciences, Cleveland, Ohio, 44195; 84 - Johns Hopkins University, Medicine, Baltimore, Maryland, 21218 ; 85 - National 
Heart, Lung, and Blood Institute, National Institutes of Health, Bethesda, Maryland, 20892; 86 - Boston University,

714 Massachusetts General Hospital, Boston University School of Medicine, Boston, Massachusetts, 02114 ; 87 - University of

715 Pittsburgh, Pittsburgh, Pennsylvania, 15260; 88 - Fundação de Hematologia e Hemoterapia de Pernambuco - Hemope,

716 Recife, 52011-000; 89 - National Jewish Health, National Jewish Health, Denver, Colorado, 80206 ; 90 - Medical College

717 of Wisconsin, Milwaukee, Wisconsin, 53226; 91 - University of Texas Health at Houston, Pediatrics, Houston, Texas,

718 77030; 92 - University of California, San Francisco, San Francisco, California, 94143; 93 - Stanford University, Biomedical

719 Data Science, Stanford, California, 94305; 94 - University of Washington, Biostatistics, Seattle, Washington, 98195 ; 95 -

720 University of Colorado at Denver, Denver, Colorado, 80204; 96 - Brigham \& Women's Hospital, Boston, Massachusetts,

721 02115; 97 - University of Montreal; 98 - Washington State University, Pullman, Washington, $99164 ; 99$ - University of

722 California, Los Angeles, Los Angeles, California, 90095; 100 - Brigham \& Women's Hospital; 101 - Brigham \& Women's

723 Hospital, Medicine, Boston, Massachusetts, 02115; 102 - National Taiwan University, Taipei, 10617; 103 - Brigham \&

724 Women's Hospital, Division of Preventive Medicine, Boston, Massachusetts, 02215; 104 - University of Virginia,

725 Charlottesville, Virginia, 22903; 105 - Lundquist Institute, Torrance, California, 90502; 106 - Cleveland Clinic, Cleveland

726 Clinic, Cleveland, Ohio, 44195; 107 - Broad Institute, Metabolomics Platform, Cambridge, Massachusetts, $02142 ; 108$ -

727 Cleveland Clinic, Immunity and Immunology, Cleveland, Ohio, 44195; 109 - University of Vermont, Burlington, Vermont,

728 05405; 110 - National Jewish Health, Denver, Colorado, 80206; 111 - Boston University, Biostatistics, Boston,

729 Massachusetts, 02115; 112 - Vitalant Research Institute, San Francisco, California, 94118; 113 - University of Illinois at 
730 Chicago, Chicago, Illinois, 60607; 114 - University of Chicago, Chicago, Illinois, 60637; 115 - Mayo Clinic, Health

731 Quantitative Sciences Research, Rochester, Minnesota, 55905; 116 - Vanderbilt University, Nashville, Tennessee, 37235;

732117 - University of Cincinnati, Cincinnati, Ohio, 45220; 118 - University of North Carolina, Chapel Hill, North Carolina,

733 27599; 119 - University of Texas Rio Grande Valley School of Medicine, Edinburg, Texas, 78539; 120 - Brown University,

734 Providence, Rhode Island, 02912; 121 - Harvard University, Channing Division of Network Medicine, Cambridge,

735 Massachusetts, 02138; 122 - National Jewish Health, Center for Genes, Environment and Health, Denver, Colorado,

736 80206; 123 - University of North Carolina, Epidemiology, Chapel Hill, North Carolina, 27599; 124 - Washington University

737 in St Louis, St Louis, Missouri, 63130; 125 - Fred Hutchinson Cancer Research Center, Seattle, Washington, 98109; 126 -

738 New York Genome Center, New York City, New York, 10013; 127 - Icahn School of Medicine at Mount Sinai, New York,

739 New York, 10029; 128 - University of Pittsburgh, Pittsburgh, Pennsylvania; 129 - Beth Israel Deaconess Medical Center,

740 Boston, Massachusetts, 02215; 130 - Boston Children's Hospital, Harvard Medical School, Department of Psychiatry,

741 Boston, Massachusetts, 02115; 131 - University of Texas Rio Grande Valley School of Medicine, San Antonio, Texas,

742 78229; 132 - Mass General Brigham, Obstetrics and Gynecology, Boston, Massachusetts, 02115; 133 - Indiana

743 University, OB/GYN, Indianapolis, Indiana, 46202; 134 - University of Mississippi, Cardiology, Jackson, Mississippi,

744 39216; 135 - University of Calgary, Medicine, Calgary; 136 - University of Maryland, Genetics, Philadelphia, Pennsylvania,

745 19104; 137 - Yale University, Department of Chronic Disease Epidemiology, New Haven, Connecticut, 06520; 138 -

746 University of Washington, Epidemiology, Seattle, Washington, 98195; 139 - Wake Forest Baptist Health, Winston-Salem, 
747 North Carolina, 27157; 140 - Brigham \& Women's Hospital, Channing Division of Network Medicine, Boston,

748 Massachusetts, 02115; 141 - University of Alabama, Birmingham, Alabama, 35487; 142 - University of Texas Health at

749 Houston, Houston, Texas, 77225; 143 - University of lowa, lowa City, lowa, 52242; 144 - National Health Research

750 Institute Taiwan, Institute of Population Health Sciences, NHRI, Miaoli County, 350; 145 - Tri-Service General Hospital

751 National Defense Medical Center; 146 - Blood Works Northwest, Seattle, Washington, $98104 ; 147$ - Taichung Veterans

752 General Hospital Taiwan, Taichung City, 407; 148 - Oklahoma State University Medical Center, Internal Medicine,

753 Dlvision of Endocrinology, Diabetes and Metabolism, Columbus, Ohio, 43210; 149 - Blood Works Northwest, Research

754 Institute, Seattle, Washington, 98104; 150 - University of Michigan, Biostatistics, Ann Arbor, Michigan, $48109 ; 151$ -

755 University of California, San Francisco, San Francisco, California, 94118; 152 - Harvard University, Cambridge,

756 Massachusetts, 02138; 153 - McGill University, Montréal, QC H3A 0G4; 154 - University of Colorado at Denver,

757 Epidemiology, Aurora, Colorado, 80045; 155 - Loyola University, Public Health Sciences, Maywood, Illinois, $60153 ; 156$ -

758 Harvard School of Public Health, Biostats, Boston, Massachusetts, 02115; 157 - Boston University, Boston,

759 Massachusetts, 02215; 158 - Brown University, Epidemiology and Medicine, Providence, Rhode Island, $02912 ; 159$ -

760 Duke University, Cardiology, Durham, North Carolina, 27708; 160 - Stanford University, Cardiovascular Institute, Stanford,

761 California, 94305; 161 - Ohio State University, Division of Pulmonary, Critical Care and Sleep Medicine, Columbus, Ohio,

762 43210; 162 - University of Texas Rio Grande Valley School of Medicine, Brownsville, Texas, 78520; 163 - Broad Institute,

763 Harvard University, Massachusetts General Hospital; 164 - George Washington University, cardiology, Washington, 
District of Columbia, 20037; 165 - University of Washington, Genome Sciences, Seattle, Washington, $98195 ; 166$ - RTI International; 167 - Massachusetts General Hospital, Medicine, Boston, Massachusetts, 02114 ; 168 - University of Arizona, Tucson, Arizona, 85721; 169 - Stanford University, Center For Sleep Sciences and Medicine, Palo Alto, California, 94304; 170 - National Institute of Child Health and Human Development, National Institutes of Health, Bethesda, Maryland, 20892; 171 - Oklahoma Medical Research Foundation, Genes and Human Disease, Oklahoma City, Oklahoma, 73104; 172 - Howard University, Washington, District of Columbia, 20059; 173 - University of Maryland, Balitmore, Maryland, 21201; 174 - University at Buffalo, Buffalo, New York, 14260; 175 - University of Pennsylvania, Division of Sleep Medicine/Department of Medicine, Philadelphia, Pennsylvania, 19104-3403; 176 - Stanford University, Stanford Cardiovascular Institute, Stanford, California, 94305; 177 - University of Minnesota, Minneapolis, Minnesota, 55455; 178 - RTI International, Biostatistics and Epidemiology Division, Research Triangle Park, North Carolina, 277092194; 179 - Fred Hutchinson Cancer Research Center, Fred Hutch and UW, Seattle, Washington, 98109 ; 180 - Johns Hopkins University, Cardiology/Medicine, Baltimore, Maryland, 21218; 181 - University of Colorado at Denver, Medicine, Denver, Colorado, 80204; 182 - University of Colorado at Denver, Denver, Colorado, 80045; 183 - University of North Carolina, Genetics, Chapel Hill, North Carolina, 27599; 184 - Northwestern University, Chicago, Illinois, $60208 ; 185$ - New York Genome Center, New York Genome Center, New York City, New York, 10013; 186 - Fred Hutchinson Cancer Research Center, University of Washington, Seattle, Washington, 98109; 187 - Lutia I Puava Ae Mapu I Fagalele, Apia; 188 - University of Ottawa, Sleep Research Unit, University of Ottawa Institute for Mental Health Research, Ottawa, ON 
K1Z 7K4; 189 - Vanderbilt University, Medicine, Pharmacology, Biomedicla Informatics, Nashville, Tennessee, 37235; 190 - Universidade de Sao Paulo, Faculdade de Medicina, Sao Paulo, 01310000; 191 - Columbia University, New York, New York, 10027; 192 - Lundquist Institute, TGPS, Torrance, California, 90502; 193 - Harvard University, Division of Hematology/Oncology, Boston, Massachusetts, 02115; 194 - Harvard Medical School, Genetics, Boston, Massachusetts, 02115; 195 - Harvard Medical School, Boston, Massachusetts, 02115; 196 - Université Laval, Quebec City, G1V 0A6; 197 - Emory University, Pediatrics, Atlanta, Georgia, 30307; 198 - Emory University, Human Genetics, Atlanta, Georgia, 30322; 199 - Vanderbilt University, Medicine/Cardiology, Nashville, Tennessee, 37235; 200 - UMass Memorial Medical Center, Worcester, Massachusetts, 01655; 201 - University of Saskatchewan, Saskatoon, SK S7N 5C9; 202 - University of Michigan; 203 - Albert Einstein College of Medicine, New York, New York, 10461; 204 - Wake Forest Baptist Health, Biostatistical Sciences, Winston-Salem, North Carolina, 27157; 205 - University of Colorado at Denver, Genomic Cardiology, Aurora, Colorado, 80045; 206 - Stanford University, Genetics, Stanford, California, 94305; 207 - University of Washington, University of Washington, Department of Genome Sciences, Seattle, Washington, 98195 ; 208 - University of Pennsylvania, Genetics, Philadelphia, Pennsylvania, 19104; 209 - University of Alabama, Biostatistics, Birmingham, Alabama, 35487; 210 - University of Washington, Department of Biostatistics, Seattle, Washington, $98195 ; 211$ University of Southern California, USC Methylation Characterization Center, University of Southern California, California, 90033; 212 - Brigham \& Women's Hospital, Mass General Brigham, Boston, Massachusetts, $02115 ; 213$ - Brigham \& Women's Hospital, Channing Division of Network Medicine, Department of Medicine, Boston, Massachusetts, 02115 ; 214 
- Massachusetts General Hospital, Boston, Massachusetts, 02114; 215 - Indiana University, Epidemiology, Indianapolis,

799 Indiana, 46202; 216 - Henry Ford Health System, Detroit, Michigan, 48202; 217 - Beth Israel Deaconess Medical Center,

800 Cardiology, Cambridge, Massachusetts, 02139; 218 - University of Pittsburgh, Medicine, Pittsburgh, Pennsylvania, 15260;

801219 - University of Michigan, Department of Epidemiology, Ann Arbor, Michigan, 48109; 220 - Case Western Reserve

802 University, Department of Population and Quantitative Health Sciences, Cleveland, Ohio, 44106

803

\section{Competing interests}

805 P.N. reports investigator-initiated grant support from Amgen, Apple, AstraZeneca, and Boston Scientific, personal fees

806 from Apple, AstraZeneca, Blackstone Life Sciences, Foresite Labs, Genentech, and Novartis, and spousal employment at

807 Vertex, all unrelated to the present work. BP serves on the Steering Committee of the Yale Open Data Access Project

808 funded by Johnson \& Johnson. MEM receives funding from Regeneron Pharmaceutical Inc. unrelated to this work. SA

809 has employment and equity in 23andMe, Inc. The spouse of CJW works at Regeneron. 


\section{References:}

1. Cohen, J. C., Boerwinkle, E., Mosley, T. H. \& Hobbs, H. H. Sequence Variations in PCSK9, Low LDL, and Protection against Coronary Heart Disease. N. Engl. J. Med. 354, 1264-1272 (2006).

2. Cohen, J. et al. Low LDL cholesterol in individuals of African descent resulting from frequent nonsense mutations in PCSK9. Nat. Genet. 37, 161-165 (2005).

3. Musunuru, K. et al. Exome Sequencing, ANGPTL3 Mutations, and Familial Combined Hypolipidemia. N. Engl. J. Med. 363, 22202227 (2010).

4. Stitziel, N. O. et al. ANGPTL3 Deficiency and Protection Against Coronary Artery Disease. J. Am. Coll. Cardiol. 69, 2054-2063 (2017).

5. Dewey, F. E. et al. Genetic and Pharmacologic Inactivation of ANGPTL3 and Cardiovascular Disease. N. Engl. J. Med. 377, 211221 (2017).

6. Manolio, T. A. et al. Finding the missing heritability of complex diseases. Nature 461, 747-753 (2009).

7. Pollin, T. I. et al. A null mutation in human APOC3 confers a favorable plasma lipid profile and apparent cardioprotection. Science 322, 1702-1705 (2008).

8. Shen, H. et al. Familial defective apolipoprotein B-100 and increased low-density lipoprotein cholesterol and coronary artery calcification in the old order amish. Arch. Intern. Med. 170, 1850-1855 (2010). 
9. Saleheen, D. et al. Human knockouts and phenotypic analysis in a cohort with a high rate of consanguinity. Nature 544, 235-239 $828 \quad$ (2017).

10. Exome Aggregation Consortium et al. Analysis of protein-coding genetic variation in 60,706 humans. Nature 536, 285-291 (2016).

831 11. Samocha, K. E. et al. A framework for the interpretation of de novo mutation in human disease. Nat. Genet. 46, $944-950$ (2014).

832 12. Natarajan, P. et al. Chromosome Xq23 is associated with lower atherogenic lipid concentrations and favorable cardiometabolic indices. Nat. Commun. 12, 2182 (2021).

834 13. Li, X. et al. Dynamic incorporation of multiple in silico functional annotations empowers rare variant association analysis of large 835 whole-genome sequencing studies at scale. Nat. Genet. 52, 969-983 (2020).

836 14. Natarajan, P. et al. Deep-coverage whole genome sequences and blood lipids among 16,324 individuals. Nat. Commun. 9, 3391 837 (2018).

838 15. Klarin, D. et al. Genetics of blood lipids among 300,000 multi-ethnic participants of the Million Veteran Program. Nat. Genet. 50, 1514-1523 (2018).

16. Hu, Y. et al. Minority-centric meta-analyses of blood lipid levels identify novel loci in the Population Architecture using

841 Genomics and Epidemiology (PAGE) study. PLoS Genet. 16, e1008684 (2020).

842 17. NHLBI Trans-Omics for Precision Medicine (TOPMed) Consortium et al. Sequencing of 53,831 diverse genomes from the NHLBI TOPMed Program. Nature 590, 290-299 (2021). 
18. Stilp, A. M. et al. A System for Phenotype Harmonization in the NHLBI Trans-Omics for Precision Medicine (TOPMed)

845 Program. Am. J. Epidemiol. (2021) doi:10.1093/aje/kwab115.

846 19. Fadista, J., Manning, A. K., Florez, J. C. \& Groop, L. The (in)famous GWAS P-value threshold revisited and updated for low-

847 frequency variants. Eur. J. Hum. Genet. EJHG 24, 1202-1205 (2016).

848 20. Purcell, S. et al. PLINK: a tool set for whole-genome association and population-based linkage analyses. Am. J. Hum. Genet. 81, $849 \quad 559-575(2007)$.

850 21. Bentley, A. R. et al. Multi-ancestry genome-wide gene-smoking interaction study of 387,272 individuals identifies new loci $851 \quad$ associated with serum lipids. Nat. Genet. 51, 636-648 (2019).

852 22. Ripatti, P. et al. Polygenic Hyperlipidemias and Coronary Artery Disease Risk. Circ. Genomic Precis. Med. 13, e002725 (2020).

853 23. van Leeuwen, E. M. et al. Meta-analysis of 49549 individuals imputed with the 1000 Genomes Project reveals an exonic 854 damaging variant in ANGPTL4 determining fasting TG levels. J. Med. Genet. 53, 441-449 (2016).

855 24. Nielsen, J. B. et al. Loss-of-function genomic variants highlight potential therapeutic targets for cardiovascular disease. Nat.

856 Commun. 11, 6417 (2020).

857 25. Aragam, K. G. et al. Limitations of Contemporary Guidelines for Managing Patients at High Genetic Risk of Coronary Artery

858 Disease. J. Am. Coll. Cardiol. 75, 2769-2780 (2020).

859 26. Park, J. et al. Exome-wide evaluation of rare coding variants using electronic health records identifies new gene-phenotype

$860 \quad$ associations. Nat. Med. 27, 66-72 (2021). 
27. Barter, P. J. et al. Effects of Torcetrapib in Patients at High Risk for Coronary Events. N. Engl. J. Med. 357, 2109-2122 (2007).

28. Schwartz, G. G. et al. Effects of Dalcetrapib in Patients with a Recent Acute Coronary Syndrome. N. Engl. J. Med. 367, 20892099 (2012).

29. The HPS3/TIMI55-REVEAL Collaborative Group. Effects of Anacetrapib in Patients with Atherosclerotic Vascular Disease. N. Engl. J. Med. 377, 1217-1227 (2017).

30. Lincoff, A. M. et al. Evacetrapib and Cardiovascular Outcomes in High-Risk Vascular Disease. N. Engl. J. Med. 376, 1933-1942 (2017).

31. Lonsdale, J. et al. The Genotype-Tissue Expression (GTEx) project. Nat. Genet. 45, 580-585 (2013).

32. Fairoozy, R. H., White, J., Palmen, J., Kalea, A. Z. \& Humphries, S. E. Identification of the Functional Variant(s) that Explain the Low-Density Lipoprotein Receptor (LDLR) GWAS SNP rs6511720 Association with Lower LDL-C and Risk of CHD. PloS One 11, e0167676 (2016).

33. Li, Z. et al. Dynamic Scan Procedure for Detecting Rare-Variant Association Regions in Whole-Genome Sequencing Studies. Am. J. Hum. Genet. 104, 802-814 (2019).

34. Roses, A. D. et al. A TOMM40 variable-length polymorphism predicts the age of late-onset Alzheimer's disease. Pharmacogenomics J. 10, 375-384 (2010).

35. Li, G. et al. TOMM40 intron 6 poly-T length, age at onset, and neuropathology of AD in individuals with APOE $83 / \varepsilon 3$. Alzheimers Dement. J. Alzheimers Assoc. 9, 554-561 (2013). 
36. Glazier, A. M., Scott, J. \& Aitman, T. J. Molecular basis of the Cd36 chromosomal deletion underlying SHR defects in insulin action and fatty acid metabolism. Mamm. Genome Off. J. Int. Mamm. Genome Soc. 13, 108-113 (2002).

37. Global Lipids Genetics Consortium. Discovery and refinement of loci associated with lipid levels. Nat. Genet. 45, 1274-1283 (2013).

38. Willer, C. J. et al. Discovery and refinement of loci associated with lipid levels. Nat. Genet. 45, 1274-1283 (2013).

39. ENGAGE Consortium et al. The impact of low-frequency and rare variants on lipid levels. Nat. Genet. 47, 589-597 (2015).

40. The Myocardial Infarction Genetics Consortium Investigators. Inactivating Mutations in NPC1L1 and Protection from Coronary Heart Disease. N. Engl. J. Med. 371, 2072-2082 (2014).

41. GLGC Consortium et al. Exome chip meta-analysis identifies novel loci and East Asian-specific coding variants that contribute to lipid levels and coronary artery disease. Nat. Genet. 49, 1722-1730 (2017).

42. Hoffmann, T. J. et al. A large electronic-health-record-based genome-wide study of serum lipids. Nat. Genet. 50, 401-413 (2018).

43. Martin, A. R. et al. Clinical use of current polygenic risk scores may exacerbate health disparities. Nat. Genet. 51, 584-591 (2019).

44. Peloso, G. M. \& Natarajan, P. Insights from population-based analyses of plasma lipids across the allele frequency spectrum. Curr. Opin. Genet. Dev. 50, 1-6 (2018).

45. Kremer, L. S. et al. Genetic diagnosis of Mendelian disorders via RNA sequencing. Nat. Commun. 8, 15824 (2017). 
46. Cummings, B. B. et al. Improving genetic diagnosis in Mendelian disease with transcriptome sequencing. Sci. Transl. Med. 9, eaal5209 (2017).

47. Genome Aggregation Database Production Team et al. Transcript expression-aware annotation improves rare variant interpretation. Nature 581, 452-458 (2020).

48. Mendes de Almeida, R. et al. Whole gene sequencing identifies deep-intronic variants with potential functional impact in patients with hypertrophic cardiomyopathy. PLOS ONE 12, e0182946 (2017).

49. Vitsios, D., Dhindsa, R. S., Middleton, L., Gussow, A. B. \& Petrovski, S. Prioritizing non-coding regions based on human genomic constraint and sequence context with deep learning. Nat. Commun. 12, 1504 (2021).

50. di Iulio, J. et al. The human noncoding genome defined by genetic diversity. Nat. Genet. 50, 333-337 (2018).

51. Genome Aggregation Database Consortium et al. The mutational constraint spectrum quantified from variation in 141,456 humans. Nature 581, 434-443 (2020).

52. Khera, A. V. et al. Diagnostic Yield and Clinical Utility of Sequencing Familial Hypercholesterolemia Genes in Patients With Severe Hypercholesterolemia. J. Am. Coll. Cardiol. 67, 2578-2589 (2016).

53. Benn, M., Watts, G. F., Tybjærg-Hansen, A. \& Nordestgaard, B. G. Mutations causative of familial hypercholesterolaemia: screening of 98098 individuals from the Copenhagen General Population Study estimated a prevalence of 1 in 217 . Eur. Heart J. 37, 1384-1394 (2016). 
54. Grundy, S. M. et al. 2018 AHA/ACC/AACVPR/AAPA/ABC/ACPM/ADA/AGS/APhA/ASPC/NLA/PCNA Guideline on the Management of Blood Cholesterol: Executive Summary. J. Am. Coll. Cardiol. 73, 3168-3209 (2019).

55. Sturm, A. C. et al. Clinical Genetic Testing for Familial Hypercholesterolemia. J. Am. Coll. Cardiol. 72, 662-680 (2018).

56. Reeskamp, L. F. et al. A Deep Intronic Variant in $L D L R$ in Familial Hypercholesterolemia: Time to Widen the Scope? Circ. Genomic Precis. Med. 11, (2018).

57. Calandra, S., Tarugi, P. \& Bertolini, S. Altered mRNA splicing in lipoprotein disorders: Curr. Opin. Lipidol. 22, $93-99$ (2011).

58.; on behalf of the ACMG Laboratory Quality Assurance Committee et al. Standards and guidelines for the interpretation of sequence variants: a joint consensus recommendation of the American College of Medical Genetics and Genomics and the Association for Molecular Pathology. Genet. Med. 17, 405-423 (2015).

59. Peloso, G. M. et al. Rare Protein-Truncating Variants in APOB, Lower Low-Density Lipoprotein Cholesterol, and Protection Against Coronary Heart Disease. Circ. Genomic Precis. Med. 12, e002376 (2019).

60. Abifadel, M. et al. Mutations in PCSK9 cause autosomal dominant hypercholesterolemia. Nat. Genet. 34, 154-156 (2003).

61. Jiang, L. et al. The distribution and characteristics of LDL receptor mutations in China: A systematic review. Sci. Rep. 5, 17272 (2015).

62. Arráiz, N. et al. Novel Mutations Identification in Exon 4 of LDLR Gene in Patients With Moderate Hypercholesterolemia in a Venezuelan Population. Am. J. Ther. 17, 325-329 (2010). 
63. Gudnason, V. et al. Identification of recurrent and novel mutations in exon 4 of the LDL receptor gene in patients with familial hypercholesterolemia in the United Kingdom. Arterioscler. Thromb. J. Vasc. Biol. 13, 56-63 (1993).

64. Goldmann, R. et al. Genomic characterization of large rearrangements of the LDLR gene in Czech patients with familial hypercholesterolemia. BMC Med. Genet. 11, 115 (2010).

65. Zuk, O. et al. Searching for missing heritability: Designing rare variant association studies. Proc. Natl. Acad. Sci. 111, E455-E464 (2014).

66. Soria, L. F. et al. Association between a specific apolipoprotein B mutation and familial defective apolipoprotein B-100. Proc. Natl. Acad. Sci. U. S. A. 86, 587-591 (1989).

67. Conomos, M. P., Reiner, A. P., Weir, B. S. \& Thornton, T. A. Model-free Estimation of Recent Genetic Relatedness. Am. J. Hum. Genet. 98, 127-148 (2016).

68. Gogarten, S. M. et al. Genetic association testing using the GENESIS R/Bioconductor package. Bioinforma. Oxf. Engl. 35, 53465348 (2019).

69. Das, S. et al. Next-generation genotype imputation service and methods. Nat. Genet. 48, 1284-1287 (2016).

70. Loh, P.-R. et al. Reference-based phasing using the Haplotype Reference Consortium panel. Nat. Genet. 48, 1443-1448 (2016).

71. Fuchsberger, C., Abecasis, G. R. \& Hinds, D. A. minimac2: faster genotype imputation. Bioinformatics 31, $782-784$ (2015).

72. Zhou, W. et al. Efficiently controlling for case-control imbalance and sample relatedness in large-scale genetic association studies. Nat. Genet. 50, 1335-1341 (2018). 
73. Pulit, S. L., de With, S. A. J. \& de Bakker, P. I. W. Resetting the bar: Statistical significance in whole-genome sequencing-based association studies of global populations. Genet. Epidemiol. 41, 145-151 (2017).

74. Han, B. \& Eskin, E. Random-Effects Model Aimed at Discovering Associations in Meta-Analysis of Genome-wide Association Studies. Am. J. Hum. Genet. 88, 586-598 (2011).

75. Chen, H. et al. Control for Population Structure and Relatedness for Binary Traits in Genetic Association Studies via Logistic Mixed Models. Am. J. Hum. Genet. 98, 653-666 (2016).

76. Chen, H. et al. Efficient Variant Set Mixed Model Association Tests for Continuous and Binary Traits in Large-Scale WholeGenome Sequencing Studies. Am. J. Hum. Genet. 104, 260-274 (2019).

77. The FANTOM Consortium and the RIKEN PMI and CLST (DGT). A promoter-level mammalian expression atlas. Nature 507, $462-470$ (2014).

78. The FANTOM Consortium et al. An atlas of active enhancers across human cell types and tissues. Nature 507, 455-461 (2014).

79. The ENCODE Project Consortium. An integrated encyclopedia of DNA elements in the human genome. Nature 489, 57-74 (2012).

80. Fishilevich, S. et al. GeneHancer: genome-wide integration of enhancers and target genes in GeneCards. Database J. Biol. Databases Curation 2017, (2017).

81. Wu, M. C. et al. Rare-variant association testing for sequencing data with the sequence kernel association test. Am. J. Hum. Genet. 89, 82-93 (2011). 
960 82. Madsen, B. E. \& Browning, S. R. A groupwise association test for rare mutations using a weighted sum statistic. PLoS Genet. 5,

$961 \quad$ e1000384 (2009).

962 83. Liu, Y. et al. ACAT: A Fast and Powerful p Value Combination Method for Rare-Variant Analysis in Sequencing Studies. Am. J.

963 Hum. Genet. 104, 410-421 (2019).

964 84. Buniello, A. et al. The NHGRI-EBI GWAS Catalog of published genome-wide association studies, targeted arrays and summary 965 statistics 2019. Nucleic Acids Res. 47, D1005-D1012 (2019).

966 85. GTEx Consortium. Genetic effects on gene expression across human tissues. Nature 550, 204-213 (2017).

967

968 


\section{Figure Legends}

970 Fig1: Overall study schematic. The analyses were conducted using the multi-ancestral TOPMed freeze8 data to 971 associate whole genome sequence variation with lipid phenotypes (i.e., LDL-C, HDL-C, TC and TG). A total of 66,329

972 samples with lipids quantified data from five ancestry groups were analyzed. Single variant GWAS were carried out using 973 SAIGE on the Encore platform using SNPs with MAC >20. Both trans-ancestry and ancestry-specific GWAS were 974 conducted. Genome-wide rare variant (MAF < 1\%) gene-centric and region-based aggregate tests were grouped and 975 analyzed using STAARtopmed. Finally, single variant and rare variant associations at Mendelian dyslipidemia genes were 976 investigated in further detail.

977 TOPMed - Trans-Omics for Precision Medicine; HDL-C - High-Density Lipoprotein Cholesterol; LDL-C - Low-Density 978 Lipoprotein Cholesterol; TC - Total Cholesterol; TG - Triglycerides; GWAS - Genome Wide Association Study; SAIGE -

979 Scalable and Accurate Implementation of GEneralized mixed model; MAC - Minor Allele Count; MAF - Minor Allele

980 Frequency; SNPs - Single nucleotide polymorphisms.

981 Fig2: Summary of single variant genome wide association. Representation of the single variant GWAS results from

982 TOPMed Freeze 8 whole genome sequenced data of 66,329 samples. Each quarter represents a different lipid

983 phenotype, and dots extending in clock-wise fashion represent variants with increasing evidence of association as noted

984 by - $\log 10$ ( $p$-value), which was truncated at 200. The outer three circles show the GWAS data from TOPMed freeze8

985 where variants binned to nominally significant ( $p$-value $\left.0.05-5 \times 10^{-07}\right)$, suggestive significant $\left(p\right.$-value $\left.5 \times 10^{-07}-5 \times 10^{-09}\right)$ 
986 and genome wide significant $\left(\mathrm{p}\right.$-value $\left.<5 \times 10^{-09}\right)$. The inner three circles compare our TOPMed results with known

987 significantly associated lipid loci and variants from the MVP summary statistics and GWAS catalog to the identified novel

988 variants and loci that are genome-wide significant from the current study, respectively.

989 TOPMed - Trans-Omics for Precision Medicine; GWAS - Genome Wide Association Study; MVP - Million Veteran

990 Program.

991 Fig3: Comparison of effects estimates for HDL-C and LDL-C among variants in the CETP locus. The color scale of

992 the data points was based on -log10 p-values from HDL-C association and the size of each data point was based on -

$993 \log 10$ p-values of LDL-C association. Variants which are genome wide significant with LDL-C are represented as

994 chromosome:position:reference allele:alternate allele.

995 HDL-C - High-Density Lipoprotein Cholesterol; LDL-C - Low-Density Lipoprotein Cholesterol.

996 Fig4: Conditional analysis of coding rare-variants from the same gene and a near-by gene. Non-coding rare variant

997 sets significantly associated with TC and TG after the conditional analysis on known variants are shown with additional

998 adjustment on rare-coding variants. The additional adjustment for rare-coding variants were carried out for the same gene

999 of the aggregate set and for certain gene aggregates (SPC24) the conditional analysis was carried out with a nearby

1000 Mendelian gene. After adjusting for rare-coding variants and known variants, EHD3 signal drops minimally, whereas

1001 signal from PCSK9 (promoter-DHS, enhancer-DHS), LDLR-loci (enhancer-DHS, SPC24 enhancer-DHS) enhances

1002 significantly. APOB1, SPC24 (enhancer-CAGE), HBB and APOE signal drops after the conditional analysis on rare-coding 
1003 variants. The different colored dots on the plot represents the conditional STAAR-O p-values when adjusting for known

1004 variants (Set1) and rare-coding variants of the same or near-by gene.

1005 STAAR - variant-Set Test for Association using Annotation information; TC - Total Cholesterol; TG - Triglycerides; CAGE

1006 - Cap Analysis of Gene Expression; DHS - DNase hypersensitivity.

1007 Fig5: Influence of common and rare variants with hypercholesterolemia. In addition to monogenic contributions from

1008 rare variants in Mendelian hypercholesterolemia genes, multiple genome-wide significant LDL-C-associated common

1009 variants also yield a polygenic basis for hypercholesterolemia. In the present work, we now identify rare non-coding

1010 variants in proximity of Mendelian hypercholesterolemia genes, specifically $L D L R$ and PCSK9, that also contribute to the

1011 genetic basis of hypercholesterolemia.

1012 LDL-C - Low-Density Lipoprotein Cholesterol

1013 
1014

\begin{tabular}{|c|c|c|c|c|c|c|c|c|c|c|c|c|c|}
\hline \multirow{2}{*}{$\begin{array}{l}\text { Associated } \\
\text { Lipid } \\
\text { Phenotype }\end{array}$} & \multirow{2}{*}{$\begin{array}{l}\text { Novel } \\
\text { variant } \\
\text { class }\end{array}$} & \multirow{2}{*}{ Variants (Gene) } & \multicolumn{3}{|c|}{ TOPMed Freeze8 (N=66329) } & \multicolumn{3}{|c|}{ MGB Biobank (N=25137) } & \multicolumn{3}{|c|}{ Penn Medicine Biobank (N=20079) } & \multicolumn{2}{|c|}{$\begin{array}{l}\text { Meta Analysis } \\
\text { (METASOFT) }\end{array}$} \\
\hline & & & $\begin{array}{l}\text { TOPMed } \\
\text { Effect } \\
\text { Estimate }\end{array}$ & $\begin{array}{l}\text { TOPMed } \\
\text { P-value }\end{array}$ & $\begin{array}{c}\text { TOPMed } \\
\text { MAF }\end{array}$ & $\begin{array}{c}\text { MGB } \\
\text { Biobank } \\
\text { Effect } \\
\text { Estimate }\end{array}$ & $\begin{array}{c}\text { MGB } \\
\text { Biobank } \\
\text { P-value }\end{array}$ & $\begin{array}{c}\text { MGB } \\
\text { Biobank } \\
\text { MAF }\end{array}$ & $\begin{array}{c}\text { Penn } \\
\text { Medicine } \\
\text { Biobank } \\
\text { Effect } \\
\text { Estimate }\end{array}$ & $\begin{array}{c}\text { Penn } \\
\text { Medicine } \\
\text { Biobank } \\
\text { P-value }\end{array}$ & $\begin{array}{c}\text { Penn } \\
\text { Medicine } \\
\text { Biobank } \\
\text { MAF }\end{array}$ & Beta & P-value \\
\hline LDL-C & $\begin{array}{l}\text { Novel } \\
\text { locus }\end{array}$ & 12:97352354:T:C & -12.439 & $4.88 \times 10^{09}$ & 0.003 & 1.055 & $8.08 \times 10^{-01}$ & 0.002 & 11.441 & $3.19 \times 10^{-01}$ & 0.001 & 2.357 & $5.62 \times 10^{-01}$ \\
\hline LDL-C & $\begin{array}{r}\text { Novel } \\
\text { variant }\end{array}$ & $\begin{array}{r}16: 56957451: C: T \\
(C E T P) \\
\end{array}$ & -1.568 & $2.88 \times 10^{-09}$ & 0.283 & -1.375 & $1.53 \times 10^{-04}$ & 0.309 & -2.35 & $1.54 \times 10^{-04}$ & 0.578 & -1.624 & $2.21 \times 10^{-07}$ \\
\hline LDL-C & $\begin{array}{l}\text { Novel } \\
\text { locus }\end{array}$ & 4:176382171:C:T & -16.086 & $2.82 \times 10^{-09}$ & 0.002 & -13.340 & $1.71 \times 10^{-01}$ & 0.001 & 4.716 & $3.52 \times 10^{-01}$ & 0.005 & 0.882 & $8.44 \times 10^{-01}$ \\
\hline TC & $\begin{array}{r}\text { Novel } \\
\text { variant }\end{array}$ & $\begin{array}{r}13: 113841051: \mathrm{T}: \mathrm{C} \\
(\text { (GAS6) }\end{array}$ & 1.731 & $1.12 \times 10^{-09}$ & 0.278 & 0.890 & $3.94 \times 10^{-02}$ & 0.304 & 0.416 & $5.50 \times 10^{-01}$ & 0.563 & 0.758 & $3.89 \times 10^{-02}$ \\
\hline TC & $\begin{array}{r}\text { Novel } \\
\text { variant }\end{array}$ & $\begin{array}{r}\text { 7:137875053:T:C } \\
\text { (CREB3L2) }\end{array}$ & -4.106 & $7.54 \times 10^{-11}$ & 0.045 & -4.755 & $1.06 \times 10^{-02}$ & 0.013 & -3.365 & $7.62 \times 10^{-03}$ & 0.118 & -3.803 & $2.69 \times 10^{-04}$ \\
\hline TG & $\begin{array}{l}\text { Novel } \\
\text { locus }\end{array}$ & 11:69219641:C:T & 0.232 & $1.98 \times 10^{-09}$ & 0.002 & -0.047 & $7.33 \times 10^{-01}$ & 0.000 & 0.202 & $7.82 \times 10^{-02}$ & 0.001 & 0.101 & $2.53 \times 10^{-01}$ \\
\hline TG & $\begin{array}{r}\text { Novel } \\
\text { variant }\end{array}$ & $\begin{array}{r}13: 107551611: \mathrm{C}: \mathrm{T} \\
(F A M 155 A)\end{array}$ & 0.052 & $6.78 \times 10^{-10}$ & 0.045 & 0.016 & $4.68 \times 10^{-01}$ & 0.014 & 0.039 & $3.26 \times 10^{-01}$ & 0.016 & 0.021 & $2.62 \times 10^{-01}$ \\
\hline
\end{tabular}


1015 Table 1. Putative novel variants identified in TOPMed and evidence for replication. Variants identified as novel after

1016 comparing with the GWAS catalog and MVP summary statistics for associations with lipid phenotypes, including LDL-C,

1017 TC, and TG. All effect estimates are in mg/dL units, except for TG which was log-transformed in analysis thereby

1018 representing fractional change. Variants are categorized as novel loci or novel variant (i.e., known locus associated with

1019 another lipid phenotype) and the genes assigned to the variants per TOPMed whole genome sequence annotations

1020 (WGSA) are listed. Data is provided for the discovery (TOPMed freeze8) and replication cohorts (MGB Biobank and Penn

1021 Medicine Biobank). Meta-analysis with the replication cohorts was carried out and the corresponding beta and p-values

1022 are provided.

1023 GWAS - Genome Wide Association Study; MVP - Million Veteran Program; LDL-C - Low-Density Lipoprotein

1024 Cholesterol; TC - Total Cholesterol; TG - Triglycerides; TOPMed - Trans-Omics for Precision Medicine; WGSA - Whole

1025 Genome Sequence Annotations. 


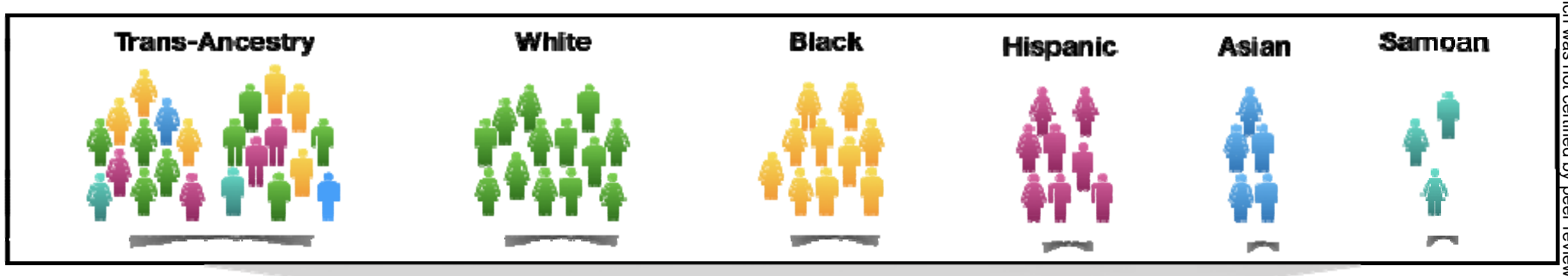
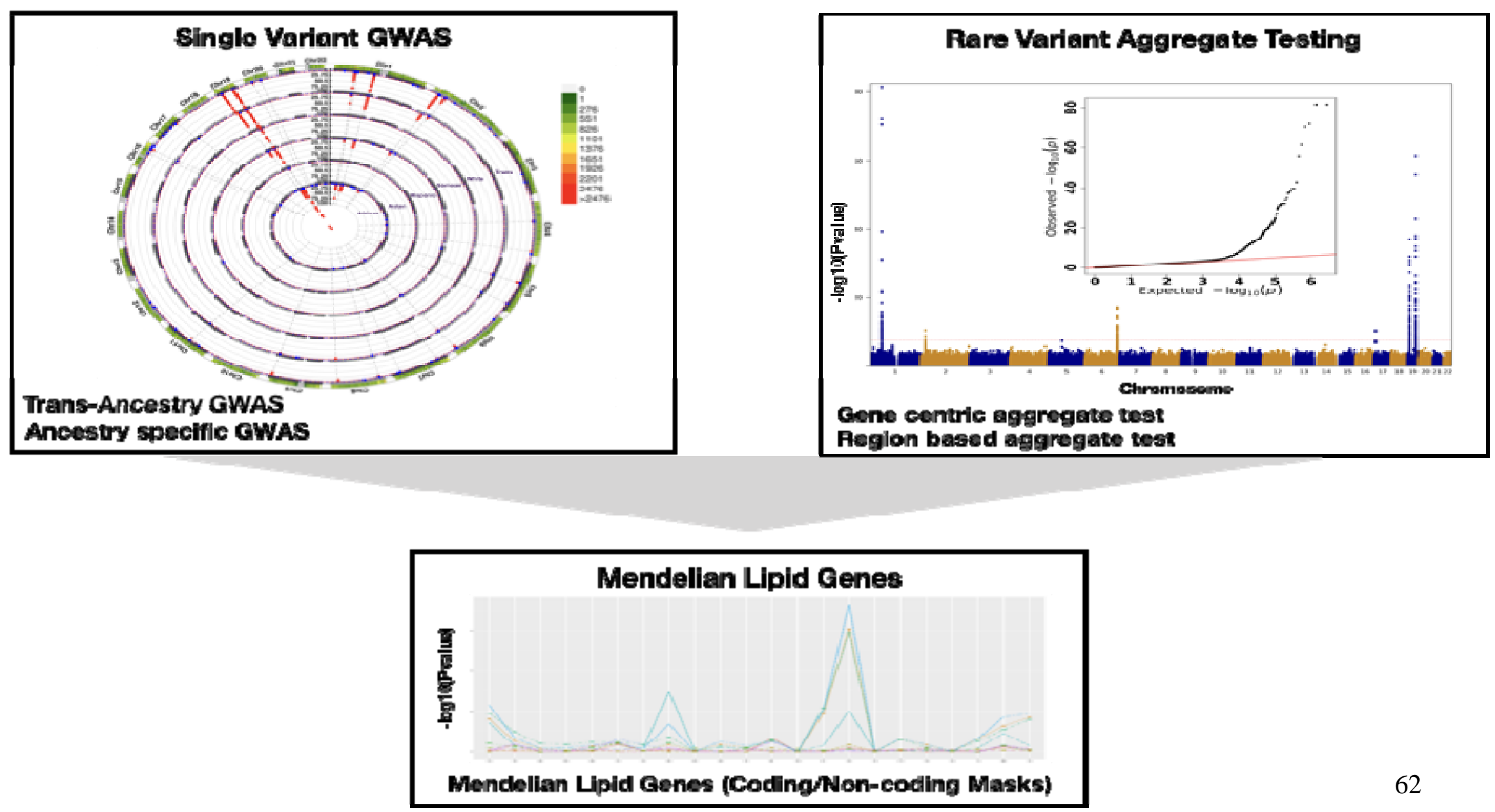

62 
1027 Fig. 1

1028 Overall study schematic. The analyses were conducted using the multi-ancestral TOPMed freeze8 data to associate

1029 whole genome sequence variation with lipid phenotypes (i.e., LDL-C, HDL-C, TC and TG). A total of 66,329 samples with

1030 lipids quantified data from five ancestry groups were analyzed. Single variant GWAS were carried out using SAIGE on the

1031 Encore platform using SNPs with MAC >20. Both trans-ancestry and ancestry-specific GWAS were conducted. Genome-

1032 wide rare variant $(\mathrm{MAF}<1 \%)$ gene-centric and region-based aggregate tests were grouped and analyzed using

1033 STAARtopmed. Finally, single variant and rare variant associations at Mendelian dyslipidemia genes were investigated in

1034 further detail.

1035 TOPMed - Trans-Omics for Precision Medicine; HDL-C - High-Density Lipoprotein Cholesterol; LDL-C - Low-Density

1036 Lipoprotein Cholesterol; TC - Total Cholesterol; TG - Triglycerides; GWAS - Genome Wide Association Study; SAIGE -

1037 Scalable and Accurate Implementation of GEneralized mixed model; MAC - Minor Allele Count; MAF - Minor Allele

1038 Frequency; SNPs - Single nucleotide polymorphisms.

1039 


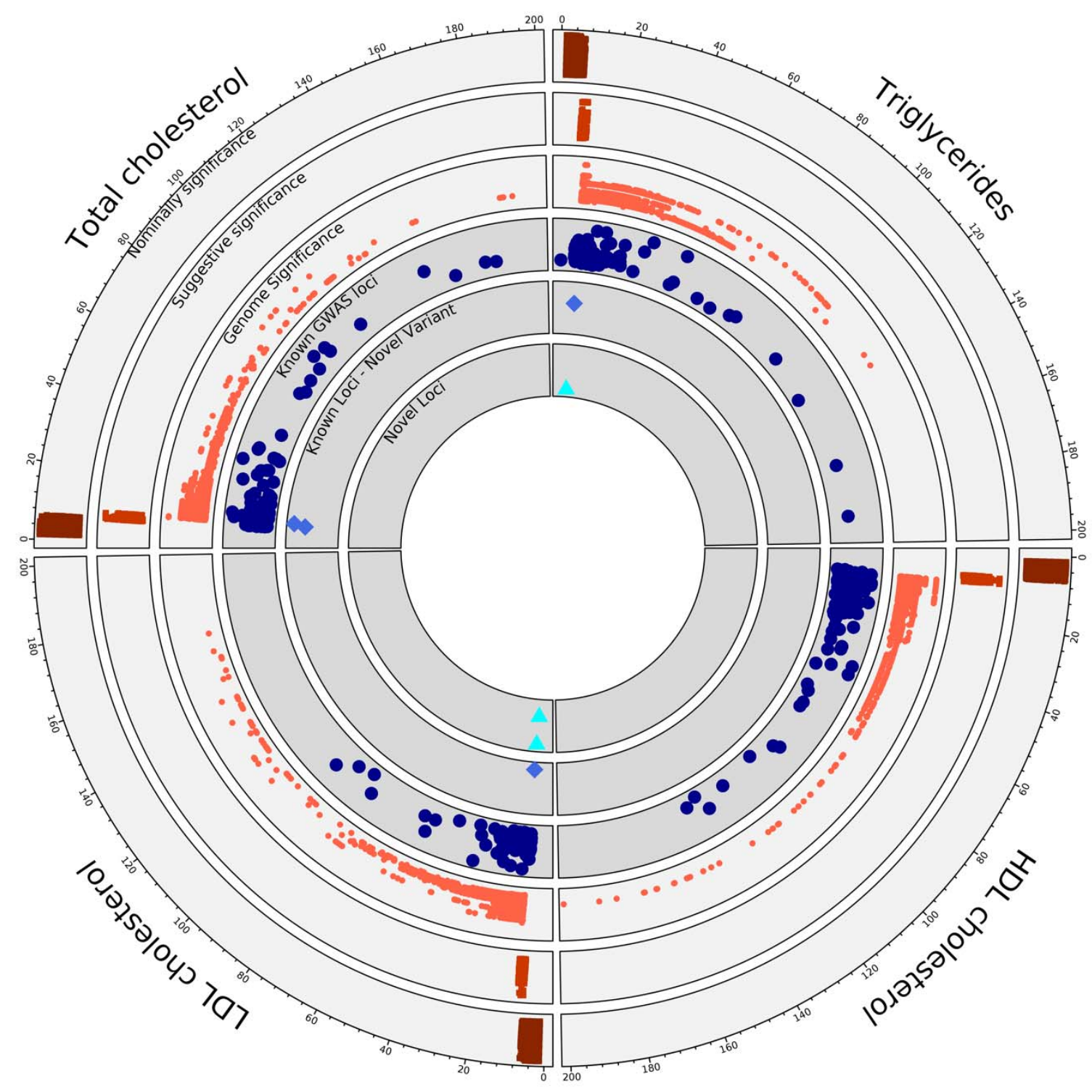

1040 
1042 Fig. 2

1043 Summary of single variant genome wide association. Representation of the single variant GWAS results from

1044 TOPMed Freeze 8 whole genome sequenced data of 66,329 samples. Each quarter represents a different lipid

1045 phenotype, and dots extending in clock-wise fashion represent variants with increasing evidence of association as noted

1046 by - $\log 10(\mathrm{p}$-value), which was truncated at 200. The outer three circles show the GWAS data from TOPMed freeze8

1047 where variants binned to nominally significant ( $p$-value $\left.0.05-5 \times 10^{-07}\right)$, suggestive significant ( $p$-value $5 \times 10^{-07}-5 \times 10^{-09}$ )

1048 and genome wide significant $\left(p\right.$-value $\left.<5 \times 10^{-09}\right)$. The inner three circles compare our TOPMed results with known

1049 significantly associated lipid loci and variants from the MVP summary statistics and GWAS catalog to the identified novel

1050 variants and loci that are genome-wide significant from the current study, respectively.

1051 TOPMed - Trans-Omics for Precision Medicine; GWAS - Genome Wide Association Study; MVP - Million Veteran

1052 Program.

1053 


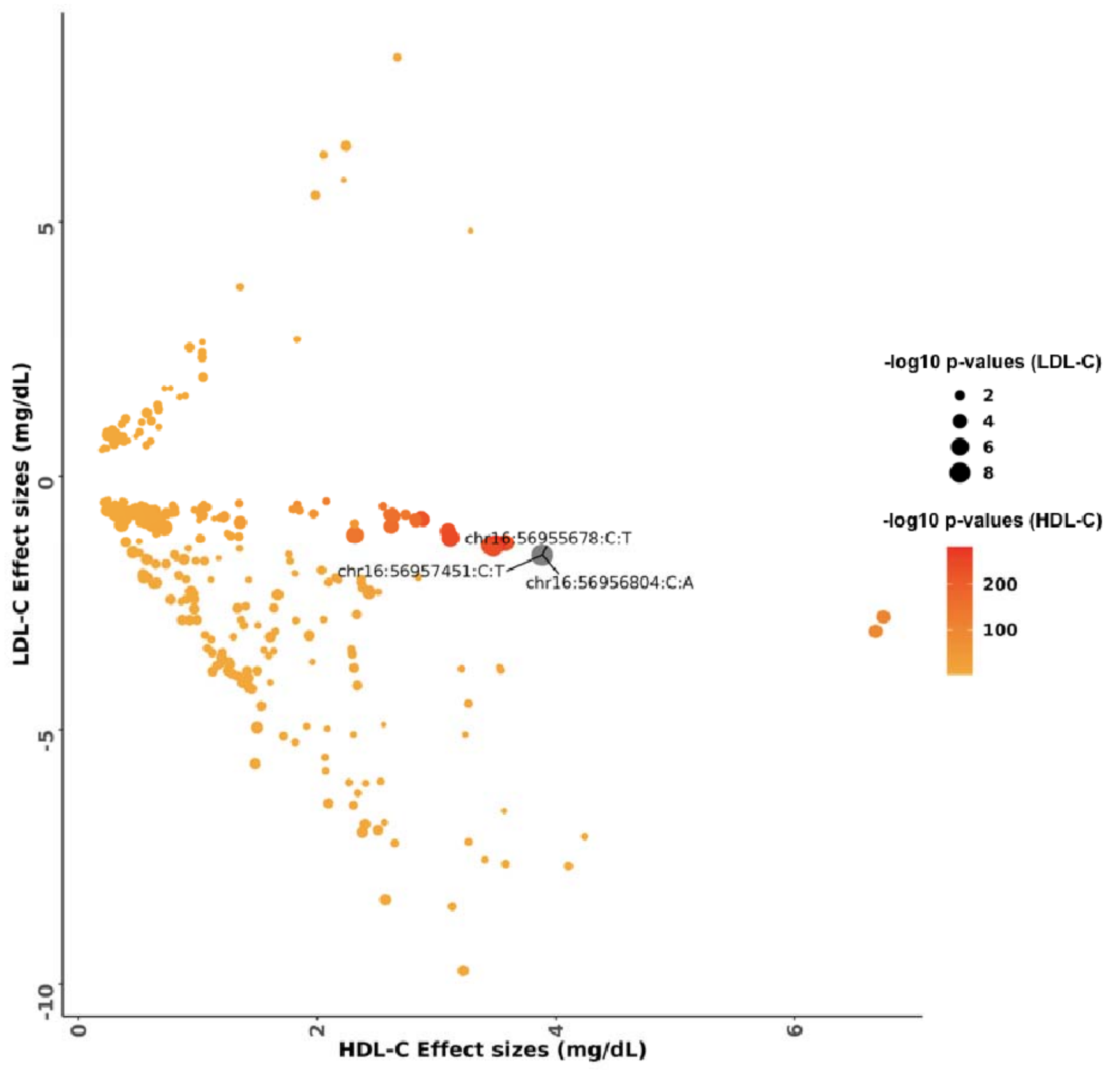


1055

1056

1057

1058

1059

1060 chromosome:position:reference allele:alternate allele.

1061 HDL-C - High-Density Lipoprotein Cholesterol; LDL-C - Low-Density Lipoprotein Cholesterol. 


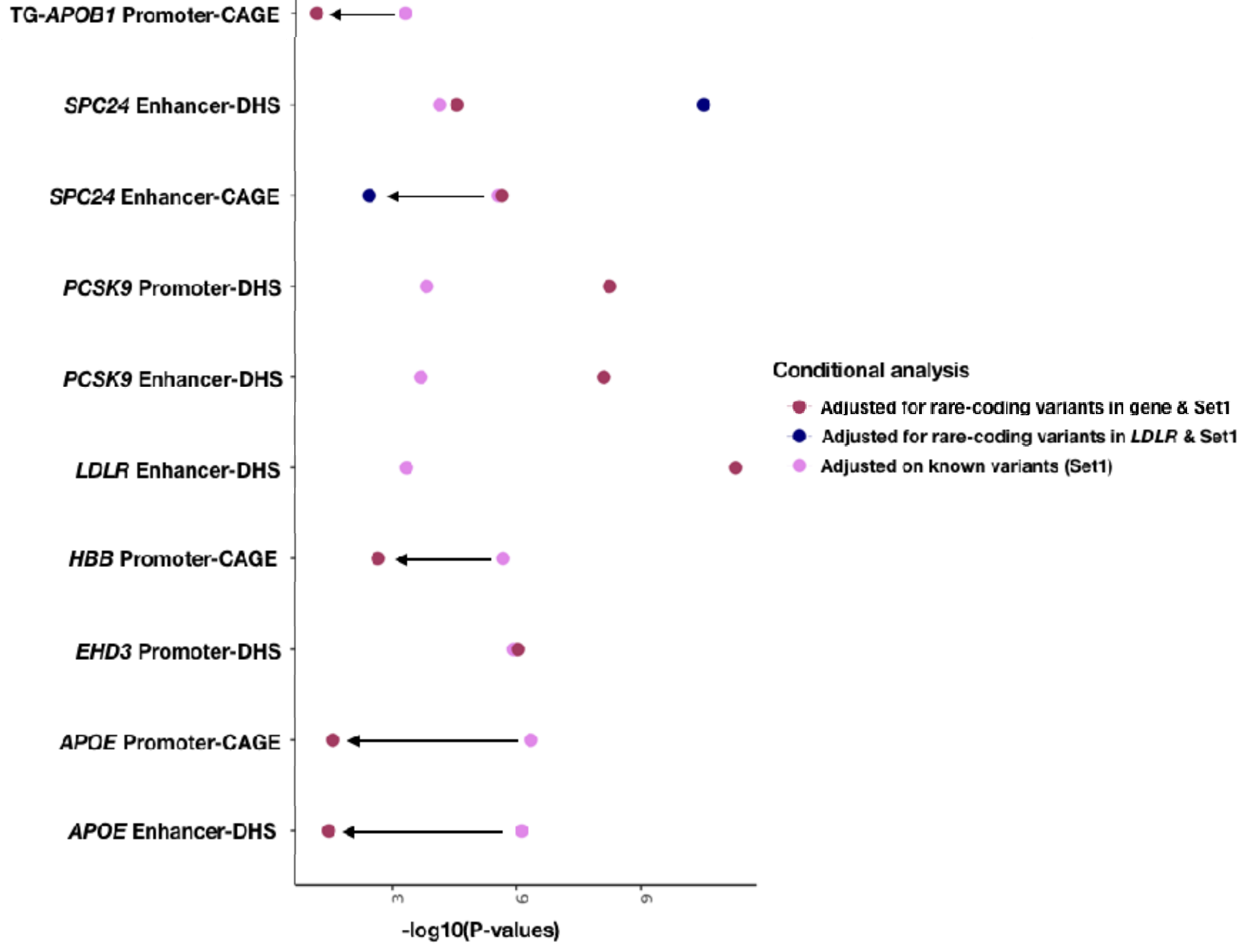


1064 Conditional analysis of coding rare-variants from the same gene and a near-by gene. Non-coding rare variant sets

1065 significantly associated with TC and TG after the conditional analysis on known variants are shown with additional

1066 adjustment on rare-coding variants. The additional adjustment for rare-coding variants were carried out for the same gene

1067 of the aggregate set and for certain gene aggregates (SPC24) the conditional analysis was carried out with a nearby

1068 Mendelian gene. After adjusting for rare-coding variants and known variants, EHD3 signal drops minimally, whereas

1069 signal from PCSK9 (promoter-DHS, enhancer-DHS), LDLR-loci (enhancer-DHS, SPC24 enhancer-DHS) enhances

1070 significantly. APOB1, SPC24 (enhancer-CAGE), HBB and APOE signal drops after the conditional analysis on rare-coding

1071 variants. The different colored dots on the plot represents the conditional STAAR-O p-values when adjusting for known

1072 variants (Set1) and rare-coding variants of the same or near-by gene.

1073 STAAR - variant-Set Test for Association using Annotation information; TC - Total Cholesterol; TG - Triglycerides; CAGE

1074 - Cap Analysis of Gene Expression; DHS - DNase hypersensitivity. 


\section{Hypercholesterolemia}

ince from monogenic rare coding variants
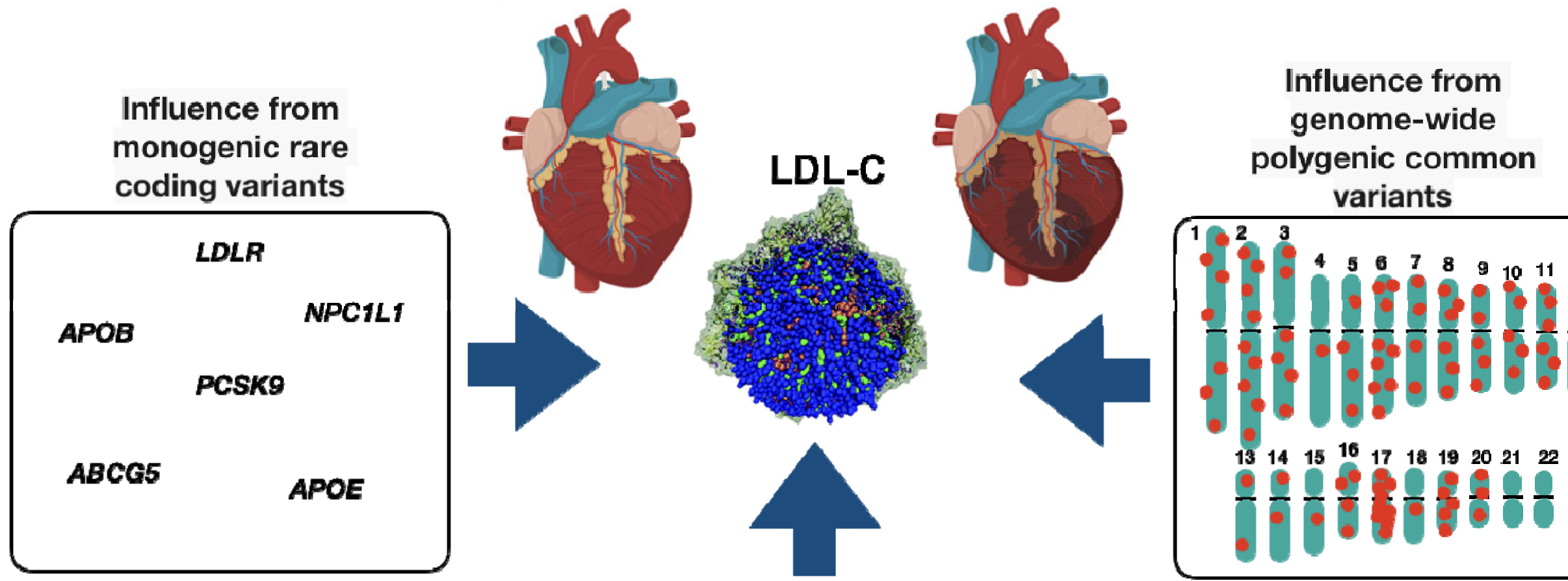

Influence from monogenic rare non-coding variants
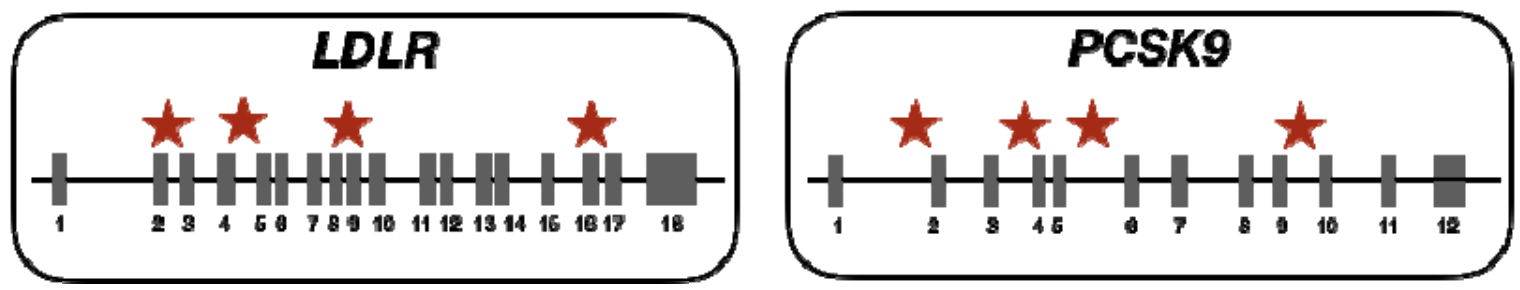


\section{$1078 \quad$ Fig. 5}

1079 Influence of common and rare variants with hypercholesterolemia. In addition to monogenic contributions from rare

1080 variants in Mendelian hypercholesterolemia genes, multiple genome-wide significant LDL-C-associated common variants

1081 also yield a polygenic basis for hypercholesterolemia. In the present work, we now identify rare non-coding variants in

1082 proximity of Mendelian hypercholesterolemia genes, specifically LDLR and PCSK9, that also contribute to the genetic

1083 basis of hypercholesterolemia.

1084 LDL-C - Low-Density Lipoprotein Cholesterol 
bioRxiv preprint doi: https://doi.org/10.1101/2021.10.11.463514; this version posted October 12, 2021. The copyright holder for this preprint (which was not certified by peer review) is the author/funder, who has granted bioRxiv a license to display the preprint in perpetuity. It is made available under aCC-BY-NC-ND 4.0 International license. 